\title{
UNEQUAL COMPRESSIVE IMAGING
}

By

\section{BETELHEM MATEOS MEKISSO}

\section{Bachelor of Science in Electrical Engineering Arba Minch University Arba Minch , Ethiopia 2007}

\author{
Submitted to the Faculty of the \\ Graduate College of \\ Oklahoma State University \\ in partial fulfillment of \\ the requirements for \\ the Degree of \\ MASTER OF SCIENCE
}

July, 2011 


\section{COPYRIGHT C}

By

\section{BETELHEM MATEOS MEKISSO}

July, 2011 


\section{UNEQUAL COMPRESSIVE IMAGING}

Thesis Approved:

Dr. Nazanin Rahnavard

Thesis Advisor

Dr. Damon Chandler

Dr. Sohum Sohoni

Dr. Mark Payton

Dean of the Graduate College 


\section{ACKNOWLEDGMENTS}

I am heartily thankful to my advisor Dr. Nazanin Rahnavard, whose encouragement, support and guidance made this thesis possible.

I would also like to extend my gratitude to all my colleagues who supported me in any respect throughout my work on this thesis. My special thanks to Ali Talari whom I had the most collaboration with.

Lastly, I would like to express my heartily appreciation to my family for their many sacrifices. 


\section{TABLE OF CONTENTS}

Chapter Page

1 INTRODUCTION 1

2 Compressive sensing and Unequal error protection 4

2.1 Compressive sensing . . . . . . . . . . . . . . . . . . 4

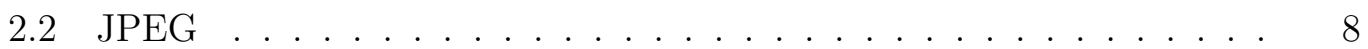

2.3 Unequal error protection with LDPC codes . . . . . . . . . . . . . . 12

2.3.1 Low-Density Parity-Check (LDPC) codes . . . . . . . . . . . . 12

2.3.2 Unequal Error Protection (UEP) . . . . . . . . . . . . . . . 13

3 Providing more protection for the Region of interest of images in $\begin{array}{lr}\text { CS } & 16\end{array}$

3.1 Region of interest of images . . . . . . . . . . . . . 16

3.2 Non-Uniform Protection for the Region of interest . . . . . . . . . . . 18

3.3 Optimal Parameter values . . . . . . . . . . . . . . . . . . . . . 21

3.4 Relationship between parameters and image quality . . . . . . . . . 25

3.5 Simulation results on sample images . . . . . . . . . . . . . . . . . 27

4 Providing more protection for images in transform basis 32

4.1 Providing more protection for the whole image . . . . . . . . . . . 32

4.1.1 The structure of transform image coefficients . . . . . . . . . 32

4.1.2 Non-uniform sampling of transform image coefficients . . . . . 34

4.1.3 Non-Uniform Sampling Implementation on Sample Images . 36

4.2 Prioritized ROI protection . . . . . . . . . . . . . . . . 41 
4.2 .1 wavelet trees . . . . . . . . . . . . . . . 42

4.2.2 Protecting ROI transform coefficients . . . . . . . . . . . 43

5 CONCLUSIONS $\quad 49$

$\begin{array}{ll}\text { BIBLIOGRAPHY } & 51\end{array}$

A Supplementary for Chapter $3 \quad 54$

B Supplementary for Chapter $4 \quad 56$ 


\section{LIST OF TABLES}

Table

Page

3.1 VIF of CS reconstruction performance employing EEP- $\Phi$ and UEP- $\Phi .28$

4.1 VIF measure of the reconstructed images employing our proposed algorithm versus TSWCS and encoding with EEP- $\Phi$. . . . . . . . 37

4.2 VIF of images reconstructed by UEP- $\phi$ for whole image, TSWCS and prioritized ROI protection algorithms. . . . . . . . . . . . . 48

A.1 VIF of CS reconstruction performance employing EEP- and UEP- $\Phi$ s for wavelet $\psi \ldots \ldots \ldots \ldots \ldots$. . . . . . . . . . . . . . . . . 54

B.1 VIF measure of the reconstructed images employing EEP- and UEP- $\Phi$ s for $\mathrm{DCT} \psi \ldots \ldots \ldots \ldots \ldots$ 


\section{LIST OF FIGURES}

Figure Page

2.1 Performance of $l_{1}$ and $l_{2}$-norms in recovering sparse signal [1]. . . . 8

2.2 visual illusion, 256 gray level shades perceived as 16 shades [2] . . . . 9

2.3 From left to right: Quantization matrix and collection process. . . . . 11

2.4 Tanner graph for the $\mathrm{H}$ matrix given above. . . . . . . . . . . . 15

2.5 Tanner graph for a code with four levels of protection. . . . . . . . . 15

3.1 Main subject detection algorithm's output. . . . . . . . . . . . . . . 17

3.2 Main subject detection and distribution of its corresponding coeffi-

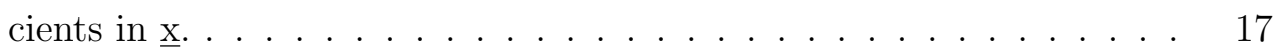

3.3 comparison of VIF for dense and sparse Gaussian matrices. . . . . . . 19

3.4 Visual performance comparison Lena images reconstructed with dense and sparse EEP- $\Phi$ s at different number of measurements. . . . . . . . 20

3.5 VIF of reconstructed Lena image at $\mathrm{m}=8192$, for different $k_{M}$ and $L$

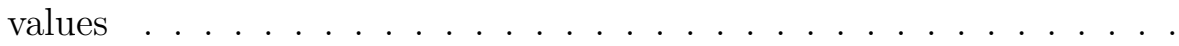

3.6 VIF of reconstructed Lena image at $\mathrm{M}=8192$ and $\mathrm{L}=16$ for different $\alpha$

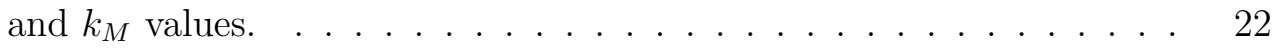

3.7 Visual performance comparison of Lena image for different $\alpha$ and $k_{M}$ values. . . . . . . . . . . . . . . . . . .

3.8 Reconstruction quality of Lena image for $k_{M}=4, \mathrm{~L}=16$ and $\alpha=0.15$ at different $M$ values. . . . . . . . . . . . . . . . . . . . . 26

3.9 Reconstruction quality of Lena image for $\mathrm{M}=8192$ and $\alpha=0.15$ at different $k_{M}$ values. . . . . . . . . . . . . . . . . . 
3.10 Reconstruction quality comparison of UEP- $\phi$ versus dense and sparse EEP- $\phi$ s for sample image PPL2. Here, $n=16384$ andm $=6000 . \quad$. .

3.11 Reconstruction quality comparison of UEP- $\phi$ versus dense and sparse EEP- $\phi$ s for sample image PPL10. Here, $n=16384 a n d \mathrm{~m}=6000 .$. .

3.12 Reconstruction quality comparison of UEP- $\phi$ versus dense and sparse EEP- $\phi$ s for sample image PPL11. Here, $n=16384 a n d \mathrm{~m}=6000 \ldots . \quad 29$

3.13 Reconstruction quality comparison of UEP- $\phi$ versus dense and sparse EEP- $\phi$ s for sample image PPL12. Here, $n=16384 a n d m=6000 . .$.

3.14 Reconstruction quality comparison of UEP- $\phi$ versus dense and sparse EEP- $\phi$ s for sample image PPL17. Here, $n=16384$ andm $=6000$. . .

3.15 Reconstruction quality comparison of UEP- $\phi$ versus dense and sparse EEP- $\phi$ s for sample image PPL20. Here, $n=16384$ andm $=6000 . .$.

4.1 Two-dimensional wavelet decomposition in Matlab . . . . . . . . . . 33

4.2 The decomposition process of the two-dimensional DWT. LPF and HPF stand for Low-Pass Filter and High-Pass Filter respectively. . . 34

4.3 Visual performance comparison of various CS image reconstructions on sample image PPL2. Here $m=4000$ and $\mathrm{L}=100 \ldots \ldots . \ldots 38$

4.4 Visual performance comparison of various CS image reconstructions on sample image PPL10. Here $m=4000$ andL $=100 \ldots \ldots 38$

4.5 Visual performance comparison of various CS image reconstructions on sample image PPL11. Here $m=4000$ andL $=100 \ldots \ldots 39$

4.6 Visual performance comparison of various CS image reconstructions on sample image PPL12. Here $m=4000$ andL $=100 \ldots \ldots 39$

4.7 Visual performance comparison of various CS image reconstructions on sample image PPL17. Here $m=4000$ andL $=100$. . . . . . . . 40

4.8 Visual performance comparison of various CS image reconstructions on sample image PPL20. Here $m=4000$ andL $=100$. . . . . . . . 40 
4.9 Image reconstruction performance comparison of our proposed algorithm versus existing algorithms. . . . . . . . . . . . . . . 41

4.10 The CPU runtime comparison of proposed algorithm and TSWCS ver-

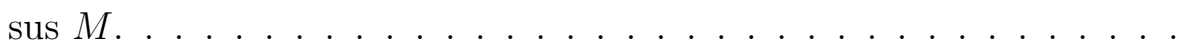

4.11 Visual performance comparison of various CS schemes for images for

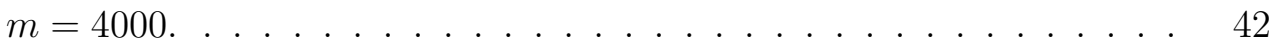

4.12 Wavelet decomposition of Lena image and wavelet tree. . . . . . . . . 43

4.13 Visual performance comparison of various CS image reconstructions for sample image PPL2. Here $m=8000$ and $\mathrm{L}=100 . \quad \ldots \ldots . . . \quad 45$

4.14 Visual performance comparison of various CS image reconstructions for sample image PPL10. Here $m=8000$ andL $=100 \ldots \ldots \ldots$

4.15 Visual performance comparison of various CS image reconstructions for sample image PPL11. Here $m=8000$ andL $=100 \ldots \ldots$

4.16 Visual performance comparison of various CS image reconstructions for sample image PPL12. Here $m=8000$ and $\mathrm{L}=100 \ldots \ldots 46$

4.17 Visual performance comparison of various CS image reconstructions for sample image PPL17. Here $m=8000$ andL $=100 \ldots \ldots . . . .47$

4.18 Visual performance comparison of various CS image reconstructions for sample image PPL20. Here $m=8000$ and $\mathrm{L}=100 \ldots \ldots . \ldots 47$

A.1 Reconstruction quality comparison of UEP- $\phi$ versus dense and sparse EEP- $\phi$ s for sample image PPL2 . . . . . . . . . . . . . . 55

A.2 Reconstruction quality comparison of UEP- $\phi$ versus dense and sparse EEP- $\phi$ s for sample image PPL10. . . . . . . . . . . . . . . 55

A.3 Reconstruction quality comparison of UEP- $\phi$ versus dense and sparse EEP- $\phi$ s for sample image PPL11. 
B.1 Visual performance comparison of various CS image reconstructions on sample image PPL2. . . . . . . . . . . . . . . . . . 57

B.2 Visual performance comparison of various CS image reconstructions on sample image PPL10. . . . . . . . . . . . . . . . . 57

B.3 Visual performance comparison of various CS image reconstructions on sample image PPL11. . . . . . . . . . . . . . . . . . . . . . 58

B.4 Visual performance comparison of various CS image reconstructions on sample images for $M=6000 \ldots \ldots \ldots$. . . . . . . . . 59

B.5 Visual performance comparison of various CS image reconstructions on sample images for $M=6000 \ldots \ldots$. . . . . . . . . . . . 60

B.6 Visual performance comparison of various CS image reconstructions on sample images for $M=6000 \ldots \ldots \ldots \ldots \ldots$

B.7 Visual performance comparison of various CS image reconstructions on sample images for $M=6000 \ldots \ldots \ldots \ldots \ldots$ 


\section{CHAPTER 1}

\section{INTRODUCTION}

Digital images have found many important applications, in various fields, since the early times when advances in digital technology were starting to be made. However, these applications were very expensive until image compression algorithms were devised [3], [2].This is due to the tremendous amount of data required to directly represent digital images which results in high storage and transmission costs.

The image compression technology finds a concise representation of digital images significantly reducing the image size. This made the application of digital images to be realizable for a wide variety of applications. The JPEG [3], for example, is a very widely used image compression algorithm up to date. The basic principle behind this image compression algorithm is that the information contained in digital images could be captured with small number of coefficients when the images are represented in a convenient basis. For this, all the coefficients of the digital image are first collected and then they are projected onto another basis on which most coefficients will be negligibly small. These negligibly small coefficients are then discarded while the original data could still be recovered accurately.

A logical question to ask here would be if it is possible to only measure the few image coefficients which capture most of the image information. This has been made possible thanks to the new field of compressive sensing (CS).

Compressive sensing only measures the informative coefficients, in sparse or com-

pressible signals, which will not be discarded later on. In other words, compressive sensing compresses digital signals while acquiring samples and hence the name com- 
pressive sensing. This significantly reduces the measurement cost and time, which would otherwise have been spent to acquire the image coefficients and their transform which would be discarded later on in the process. This is very important as data collection is very expensive in certain applications.

A very good example of applications where compressive sensing significantly reduces the measurement time is MRI. MRI normally requires a significant amount of time to acquire enough samples for producing a good quality image [4]. This is however very inconvenient for real time application as people who need an MRI examination have to wait in the MRI device for the amount of time required to take enough measurements. CS has been shown to provide very good images with less than half the number of measurements required in conventional data acquisition methods and hence significantly reducing the time required to acquire samples.

As it has been mentioned above, one of the most important characteristics of CS is that it requires significantly smaller number of samples to reconstruct a digital signal. This is especially very useful for image applications due to the huge size of typical images. Several algorithms have been devised to enhance the basic compressive sensing algorithms by exploiting the common structure of signals so as to further reduce the required measurement cost [5], [6]. Most previous works have however focused on the design of CS reconstruction algorithm. This thesis proposes two algorithms for the encoding stage of CS. These algorithms are mainly inspired by a previous work by Rahnavard et al. on unequal error protection rateless codes [7].

The first algorithm proposed for the encoding stage of CS exploits the known structure of transform image coefficients. This algorithm is shown to significantly reduce measurement cost required by state of the art CS algorithms, in terms of the required number of measurements and measurement time. The proposed algorithm makes use of the unequal error protection (UEP) principle, which is widely used in the area of error control coding, to provide more protection to the more informative 
image coefficients as compared to the others. The proposed algorithm has been tested on a number of standard images and has been shown to provide superior quality of reconstructed images when compared with state of the art CS algorithms, for the same number of measurements. The proposed algorithm is also shown to require much less CPU time when compared with other model based CS algorithms, as a result of its lower computational complexity.

In most applications involving digital images, a particular part of the image is of a higher importance than the rest of the image. An example application is face image. "When browsing a digital photograph album it is often the case that we are looking for, or most interested in, the people/faces in those photographs." The JPEG 2000 has been developed with a ROI (Region of interest) coding functionality for this reason [8]. The ROI coding of the JPEG 2000 allows the recovery of the part of an image that has been identified to be of more importance (the region of interest) than the rest of the image. To the best of our knowledge, no work has been done to provide better reconstruction quality for the more important part of a given image in the CS technology. This thesis proposes a second algorithm which exploits the UEP principle to recover the more important part of an image with more quality while the rest part of the image is not significantly degraded. The proposed algorithm is shown to be successful for both CS settings where images are represented in the spatial and transform domains. 


\section{CHAPTER 2}

\section{Compressive sensing and Unequal error protection}

\subsection{Compressive sensing}

Conventional data acquisition mechanisms rely on the famous Nyquist theorem. This theorem dictates that sampling rate of a signal should be at least twice the maximum frequency present in the signal. A new sampling theorem has recently been developed which asserts that certain signals could be recovered from much fewer number of samples than what is required by Nyquist theorem. This new sampling technique is known as compressive sampling (compressive sensing).

Compressive sampling technique is based on the fact that the information contained in certain signals is much smaller than the sampling size dictated by the signal size. This fact has been used in conventional image compression algorithms such as JPEG.

The conventional image compression algorithms first manipulate the signal so as to identify the most informative image coefficients. Then these informative coefficients are stored while the others are discarded without causing significant perceptual loss. The image is thus represented with fewer bits in this way and hence compressed. Compressive sampling, however, attempts to sample only those significant coefficients. As a result, compressive sampling compresses the signal while sampling and hence the name compressive sampling. The working principle of the compressive sensing is discussed with further detail in this section. The JPEG is also discussed further, in the next section, as an example of the conventional image compression algorithms.

One requirement to guarantee the successfulness of compressive sensing is that 
the signal must exhibit a property known as sparsity. A signal is sparse (exhibit the sparsity property) if it has only few non-zero coefficients or if most of its coefficients are very close to zero and only a few are large. More formally, Sparsity expresses the idea that the 'information rate' of a continuous time signal may be much smaller than suggested by its bandwidth, or that a discrete-time signal depends on a number of degrees of freedom which is comparably much smaller than its (finite) length.

Sparse signals could be effectively represented with a small amount of data, which contains the information of the sparse signal in a condensed form. Interestingly, most natural signals which appear to be dense in the basis in which they are acquired have a sparse representation in some other suitable basis. A very good example of such signals are images. Most natural images are dense in the spatial domain but sparse in the wavelet and DCT bases.

When images are represented in wavelet or DCT basis, $\psi$, most image coefficients will be small while only a few coefficients are large. Most of the information is, however, captured in these few large coefficients. The image could, hence, be represented with these large coefficients without much perceptual loss. This is the principle which the JPEG and JPEG 2000 image compression standards are based on. From this, it is also intuitive that the sparsity of a signal determines the degree to which a signal could be compressed. The reason why a signal must exhibit a sparsity property for CS to be successful should also be clear now. It is because, when a signal is sparse, the signal information could be acquired without much perceptual loss by sampling only the few large coefficients. CS further shows another implication of sparsity which is that it has significant bearings on the acquisition process itself and that it determines how efficiently one can acquire signals non adaptively.

Randomness is the sampling mechanism which is proposed as a nonadaptive signal acquisition method in the ground braking CS papers. As signals have to be sparse for CS technology to be successful, there is another useful property that must be 
exhibited by the sensing/sampling modality. This property is known as Incoherence.

Incoherence extends the duality between time and frequency and expresses the idea that objects having a sparse representation in $\psi$ must be spread out in the domain in which they are acquired[9]. There are normally two bases, $\phi$ and $\psi$, in the CS setting. The basis $\phi$ is used to sense the signal while the basis $\psi$ is used to represent the signal. The incoherence principle dictates that "unlike the signal of interest, the sampling/sensing waveforms have an extremely dense representation in $\psi$. "[9].

Assuming a pair of orthobasis $\phi$ and $\psi$ of size $n$, coherence could formally be defined as follows:

$$
\mu(\phi, \psi)=\sqrt{n} \cdot \max _{1 \leq k, j \leq n}\left|\left\langle\phi_{k}, \psi_{j}\right\rangle\right|
$$

Coherence measures the maximum correlation between the entries of $\phi$ and $\psi$. The coherence will, hence, be large when the entries of $\phi$ and $\psi$ are correlated and it will be small when their entries are not correlated. As $\mu(\phi, \psi) \in[1, \sqrt{n}]$ the coherence measure will be bounded within this range. CS requires the coherence between these orthobasis to be small (incoherent).

Several basis pairs have been studied and shown to be incoherent. To give a few examples, the time and frequency domains have been shown to have maximum incoherence ( (i.e. $\left.\mu^{2}(\phi, \psi)=1\right)$. Noiselet measurement matrices, $(\phi)$ have been shown to be incoherent with wavelets, spikes and Fourier basis. And more importantly, Random measurement matrices, $\phi$, were shown to be incoherent with any basis $\psi$. By extension, random waveforms with independent identically distributed (i.i.d.) entries, e.g., Gaussian or \pm 1 binary entries, will also exhibit a very low coherence with any fixed representation $\Psi$.

Ideally $n$ samples would have to be measured to successfully reconstruct a signal of length $n$. However, CS makes it possible to successfully reconstruct the signal with far less number of measurements (given the requirements for the successfulness of CS, discussed above, are met). Let the signal of length $n$ be sparse with only $k$ significant 
coefficients (k-sparse). CS could recover this signal with $m$ number of measurements with $m<<n$. More precisely:

$$
m \geq C \cdot \mu^{2}(\phi, \psi) \cdot K \cdot \log n
$$

where $\mathrm{C}$ is a positive constant. From this equation, it is clear that smaller number of measurement $(m)$ is required when incoherence is larger. And the smallest number of measurement $(m)$, which is on the order of $K \log n$, is sufficient for successful CS reconstruction when the incoherence is close to the maximum value(i.e. $\left.\mu^{2}(\phi, \psi)=1\right)$.

With sparsity and incoherence in mind, the signal acquisition process of CS is a standard setup where the signal needed to be acquired is measured with the measurement matrix $\phi$ as follows:

$$
\underline{y}=\phi \underline{x}
$$

where $x$ is the signal needed to be acquired and this signal is sparse in basis $\psi(\underline{x}=\psi \underline{s})$ where $s$ is the sparse representation of signal $x$ in $\psi$.

An important point to note about the CS acquisition process is that the measurement is signal independent (non-adaptive) as randomness is the sensing mechanism. Further more, a k-sparse signal could be reconstructed with any $m$ set of measurements, where $m$ is on the order of $K \log n$, and one does not have to choose these samples carefully. "Almost any set of this size will work [9]".

The next question to be addressed is, obviously, how to approximate the signal $\underline{x}$ (of length $n$ ) from the information captured by $\underline{y}$ (of length $m$ ). This is an ill-posed problem as $m<<n$ resulting in under determined linear system of equations. The ground breaking papers in CS $[1,10,11,12]$ propose the use of $l_{1}$-norm minimization to solve this linear problem: i.e.

$$
\underline{\hat{s}}=\operatorname{argmin}\|\underline{s}\|_{1}, \text { s.t. } \underline{y}=\phi \psi \underline{s},
$$

where $\|\underline{s}\|_{1}=\sum_{i=1}^{N}\left|s_{i}\right|$. Finally, the reconstructed image is obtained by $\underline{\hat{x}}=\psi \underline{\hat{s}}$. What this minimization means is that, of all the possible coefficient sequences(possible 
$\hat{s} \mathrm{~s})$ that satisfy $y=\phi \psi \hat{s}$, the sparsest $\hat{s}$ is chosen. When the signal is sparse enough, it has been proved that the $l_{1}$-norm minimization provides exact recovery when measurements on the order of $K \cdot \log n$ are taken.

The use of $l_{2}$-norm instead of the $l_{1}$-norm was studied in [1]. i.e. $\min \|\hat{s}\|_{l_{2}}$ so that $y=\phi \psi \hat{s}$ assuming $\|s\|_{l_{1}}=\sum_{i=1}^{n}\left|s_{i}\right| \hat{2}$. This equation finds the minimum energy $\hat{s}$ as opposed to the sparsest $\hat{s}$ that is obtained by the $l_{1}$-norm minimization. The results of the study in [1] showed that the results from the $l_{2}$-norm don't find the sparse signal accurately but rather result in many small non-zero coefficients that are not found in the original signal.
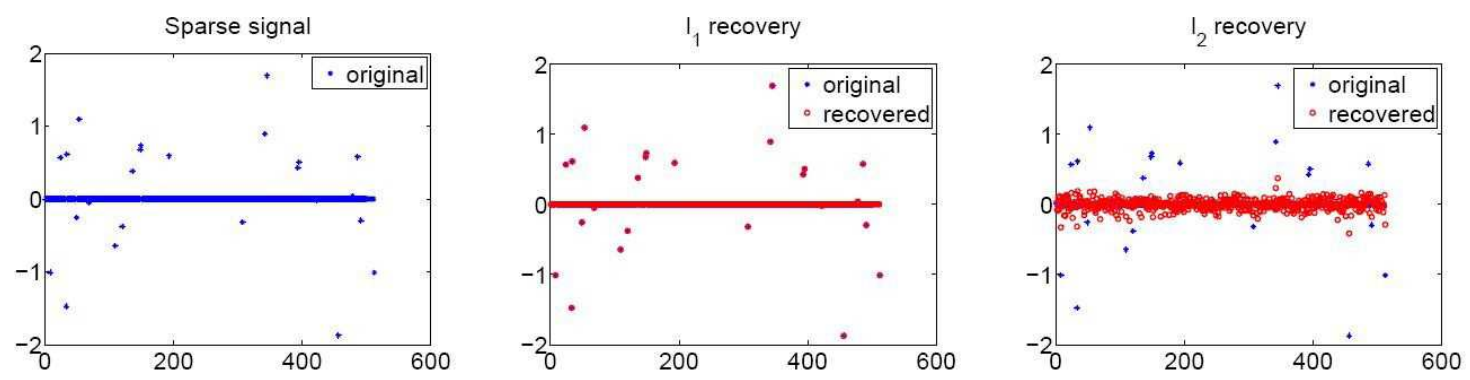

Figure 2.1: Performance of $l_{1}$ and $l_{2}$-norms in recovering sparse signal [1].

Although $l_{1}$ minimization is the most popular CS reconstruction algorithm implemented widely for CS applications, many other CS reconstruction algorithms have been proposed. Basis pursuit, matching pursuits, method of frames, best orthogonal basis and reconstruction algorithms proposed in [6] and [5] are some of the well known CS reconstruction algorithms.

\subsection{JPEG}

The word JPEG stands for Joint Picture Experts Group which was formed to solve the problem of large file sizes. This group came up with a lossy image compression 
algorithm which was named as JPEG. JPEG is designed in such a way that the loss is not visually perceptible. Furthermore, JPEG allows users to adjust the image quality or extent of compression (image size) as desired. Intuitively, the more the desired image quality the larger the images size (smaller compression).

Although JPEG works for both gray level and colored images, only the gray level case is described here to make the comparison easier as this thesis work mainly makes use of gray level images. It was first observed that the human eye could hardly perceive the difference between similar intensities. Interestingly, most natural images have very gradual change in intensity. This could be well understood from Figure 2.2 below which gives the 256 gray level shades even though the human eye perceives the image as having only 16 shades which correspond to the rows shown in the figure [2].

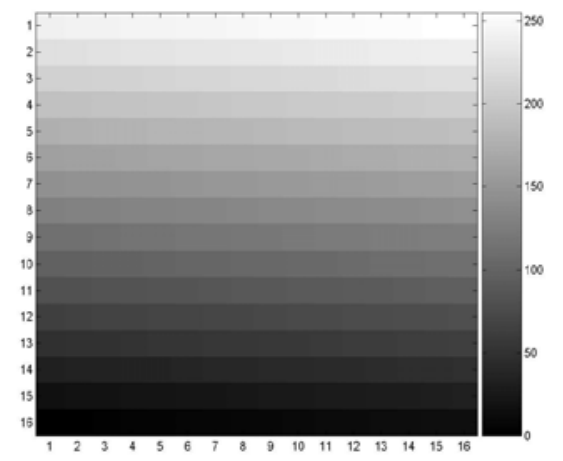

Figure 2.2: visual illusion, 256 gray level shades perceived as 16 shades [2].

From this, it is evident that one can replace the gray shades in each row with a single value and store them as a single value. This is the basic idea for JPEG compression, i.e it is sufficient to only store the gradual change in intensity instead of each pixel value hence compressing the image.

It is well known that most signals and especially images have a concise representation when represented in the frequency domain. The DCT transform, which 
transforms a signal in the spatial domain into a frequency domain, is used for JPEG. In the spatial domain, the image is represented with pixels and each pixel represents the luminosity (shade) at a particular position in the image. On the other hand, when the image is represented in the frequency domain, the entries show how the the luminosity changes across the image. Large entries show that the luminosity is changing rapidly while small entries show the luminosity is changing gradually. The DCT transform further goes additional step and separates (sorts) frequencies once they have been computed. The step by step procedure for JPEG compression is discussed below.

The first step for the JPEG compression is breaking down the image into blocks of $8 \times 8$ matrices. A two-dimensional DCT is then carried out on each of these small blocks. This process outputs a matrix of 8-bit frequency values which is of the same size as the original (i.e. $8 \times 8$ ). In this outputted matrix, the high frequencies are located at the upper left section of each small matrix.

Once the output of the DCT transform is computed, the next step is quantization. This is a process where each frequency in each small matrix is divided with the corresponding entry from a quantization matrix. The quantization matrix is also an $8 \times 8$ matrix. This quantization matrix determines how far apart the intensity levels should be spaced. As mentioned earlier, the human eye is sensitive to large luminance changes which are found at the top left corner of the small blocks. Hence, the quantization table has small entries at the top left and large entries at the bottom right. JPEG recommends certain quantization matrices that are developed based on experiments with human visual system. Users could however specify their own quantization matrices as desired. A quantization matrix given in JPEG standard is shown in Figure 2.3(a).

The entries of the matrices resulting from the quantization process are then rounded off to the nearest integer. This rounding off is clearly a lossy process es- 


$\left[\begin{array}{cccccccc}16 & 11 & 10 & 16 & 24 & 40 & 51 & 61 \\ 12 & 12 & 14 & 19 & 26 & 58 & 60 & 55 \\ 14 & 13 & 16 & 24 & 40 & 57 & 69 & 56 \\ 14 & 17 & 22 & 29 & 51 & 87 & 80 & 62 \\ 18 & 22 & 37 & 56 & 68 & 109 & 103 & 77 \\ 24 & 35 & 55 & 64 & 81 & 104 & 113 & 92 \\ 49 & 64 & 78 & 87 & 103 & 121 & 120 & 101 \\ 72 & 92 & 95 & 98 & 112 & 100 & 103 & 99\end{array}\right]$

(a) Quantization matrix.

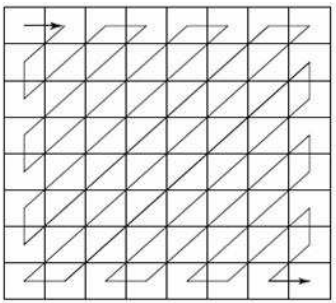

(b) Collecting entries of matrices after quantization.

Figure 2.3: From left to right: Quantization matrix and collection process.

pecially as the exact outputs of DCT are not integers in general. The matrices that result after the rounding off process are sparse where the high frequency, which correspond to the areas in the image whose change the human eye is not sensitive to, are rounded off to zeros. The quantization process could be expressed mathematically as:

$$
\text { Round }\left(\frac{8 \times 8 \text { DCT block }}{\text { quantization table }}\right)=\text { sparse matrix }
$$

Once each small matrix is quantized to output such a sparse matrix, it is represented as a single column with 64 entries. This is done in a systematic way so that the zero entries will be placed continuously at the bottom of the column. For this, each single column is formed by collecting the entries of the small matrix in a zig-zag order as shown in Figure2.3.

The Huffman or arithmetic coding is finally used to remove the zeros and encode only the important information of the block. Each small matrix is coded separately in this way. It is interesting to note that this process of coding is lossless and hence does not affect the image quality. 


\subsection{Unequal error protection with LDPC codes}

\subsubsection{Low-Density Parity-Check (LDPC) codes}

Unequal error protection (UEP) has been an area of extensive research in the field of error control coding. Error control coding is a sub-field of information theory which is concerned with reducing the bit error rates of communications over noisy channels. This error reduction is achieved by coding the information prior to transmission in such a way that it would be possible to detect and correct errors at the receiver end.

One of the most popular codes which allow error correction are linear block codes. These codes first divide the information bits into several blocks of same length $k$. Let $u=\left[u_{1} u_{2} u_{3} \ldots u_{k}\right]$ represent a single block of information also known as information word. This information word is mapped to a longer block of length $n, v=\left[v_{1} v_{2} v_{3} \ldots v_{n}\right]$ known as codeword where $n>k$. The fact that the codeword is longer than the information word shows that there exists some redundancy in the codeword.

The mapping from the information word to the codeword is a linear mapping that could be represented as: $\underline{v}=\underline{u} G$ where $\mathrm{G}$ is a $k \times n$ matrix known as generator matrix. A linear code can also be defined by its parity-check matrix $H=\left[h_{i, j}\right]$. This parity-check matrix $H$ has a size of $(n-k) \times n$ and is characterized by having rows that are orthogonal to any given row of the generator matrix $G$. Mathematically, $G H^{T}=0$.

For Low-Density Parity-Check (LDPC) codes, the parity-check matrix has a low density (very sparse) and hence the name LDPC. In the same way, a codeword $v$ is a valid codeword if and only if $H v^{T}=0$. Each row of $H$ corresponds to the parity equation while each column of $H$ corresponds to a single bit of the codeword.

LDPC codes could also be represented by Tanner graph (bipartite graph). The bipartite graph has two types of nodes known as variable (message) nodes and check nodes. The edges in the bipartite graph may only connect a variable node with a 
check node and the degree of a node is determined by the number of edges connected to it. In the bipartite graph, the variable nodes represent bits of the codeword while the check nodes represent the constraints of the parity-check. Furthermore, each edge of the graph corresponds to a 1 in the parity-check matrix. In other words, the columns of the parity-check matrix determine the connections of variable nodes while the rows determine the connections of the check nodes. Hence, the $i^{\text {th }}$ variable node is connected with the $j^{\text {th }}$ check node if and only if $h_{i, j}=1$. Figure 2.4 shows a tanner graph for a code represented by the $H$ matrix given as:

$$
H=\left[\begin{array}{llllllll}
0 & 1 & 0 & 0 & 1 & 0 & 1 & 1 \\
1 & 0 & 1 & 1 & 0 & 0 & 0 & 0 \\
1 & 0 & 1 & 1 & 0 & 0 & 0 & 0 \\
0 & 1 & 1 & 0 & 0 & 0 & 1 & 0 \\
0 & 0 & 1 & 0 & 0 & 1 & 1 & 0
\end{array}\right]
$$

\subsubsection{Unequal Error Protection (UEP)}

Providing unequal error protection (UEP) for information bits based on LDPC coding has been studied in the field of error control coding. Several different methods have been proposed to provide UEP property for LDPC codes. One of these methods is based on the irregularity of degree distribution of variable and check nodes.

It is clear that a variable node estimates its value more accurately when it has a higher degree. This is because higher degree for a variable node means that it gets more information from its neighboring check nodes. On the other hand, smaller degree is preferred for check nodes. This is because the lower the degree of a check node, the more valuable is the information the check node transmits to its neighboring variable nodes. This lays the foundation of UEP based on irregularity of degree distribution of variable and check nodes.

Let us consider a case where four different levels of protection are desired for the 
variable nodes. Assuming the same degree for all the check nodes, the degrees to the variable nodes are allocated in such a way that more edges are provided for variable nodes to which more protection is desired. Figure 2.5 shows a schematic diagram for the case where there are four protection levels.

The variable nodes are first classified into four classes and assigned different degrees $\left(\mathrm{d}_{v c 1}, d_{v c 2}, d_{v c 3}, d_{v c 4}\right)$, with variable nodes in each class having the same degree. Figure 2.5 demonstrates a scenario where the required level of protection is assumed to be highest for class 1 and decreases for the succeeding classes with class 4 having the lowest protection. As a more protected class has to have more variable node degree than a less protected one, the degree distribution will be as shown in Figure 2.5 (i.e. $d_{v c 1}>d_{v c 2}>d_{v c 3}>d_{v c 4}$ ). In this way, it has been shown that UEP codes could recover the more important classes with lower error rate while its overall error rate is competent to other codes. 


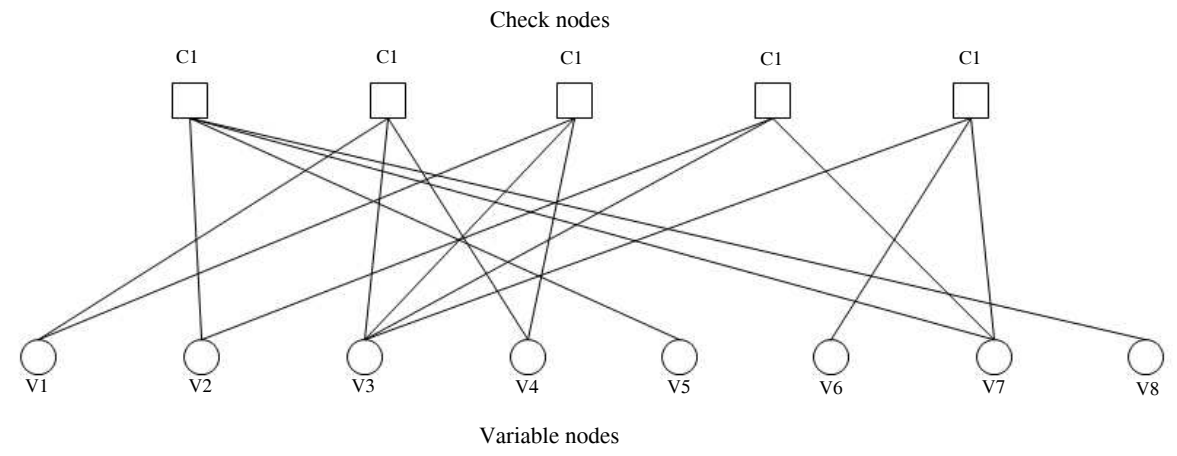

Figure 2.4: Tanner graph for the $\mathrm{H}$ matrix given above.

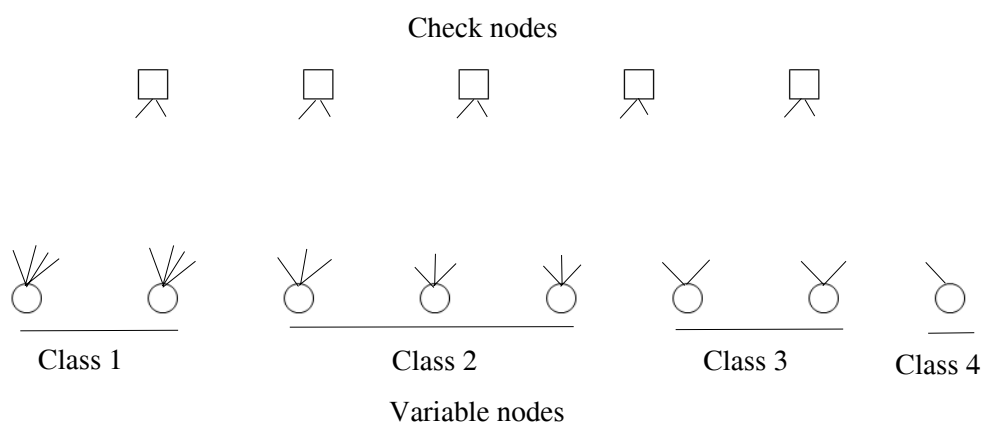

Figure 2.5: Tanner graph for a code with four levels of protection. 


\section{CHAPTER 3}

\section{Providing more protection for the Region of interest of images in CS}

In this section, we consider the scenario where a particular part of a given image is considered to be more important than the rest. An algorithm is proposed to recover the more important part of the image with more quality. For this, the more important coefficients (MICs) of the image have to be identified first.

\subsection{Region of interest of images}

In a given imagery, a certain part of the image is normally the center of interest. For example, Viewers' attention is naturally drawn to the face part of images when browsing pictures in a digital album. Although identifying the main subject in an image is a very simple task for humans, Main Subject Detection (MSD) is a difficult task for computers. As a result, different algorithms have been developed which identify the main subject in a given imagery [13].

A novel main subject detection algorithm proposed in [13] is adopted in this thesis in order to identify the main subject of images. Figure 3.1 illustrate the output of this main subject detection algorithm [13] on sample images from Microsoft Research in Cambridge ${ }^{1}$. (All images have been resized to size of $128 \times 128$ and converted to gray level for convenience.) In Figure 3.1, the part of the images identified as main subject is shown bounded with rectangles.

As images could be viewed as a matrix of pixels and as the MICs are the image coefficients corresponding to the main subject, the location of the MICs is the location

\footnotetext{
${ }^{1}$ available at http://research.microsoft.com/en-us/projects/objectclassrecognition/
} 


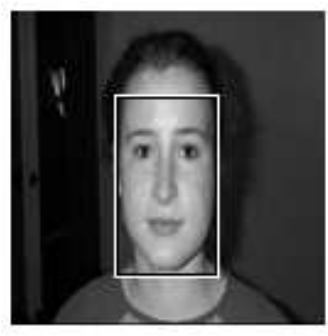

(a) PPL2.

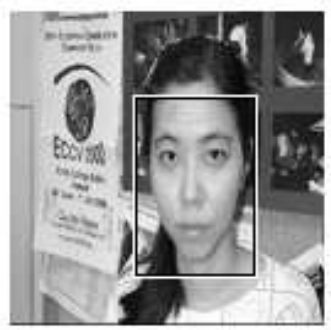

(d) PPL12.

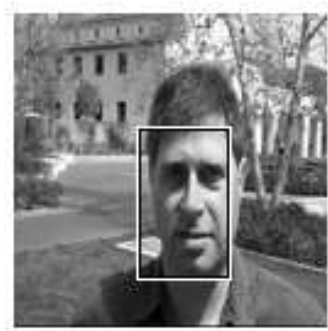

(b) PPL10.

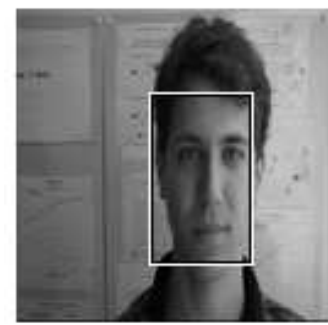

(e) PPL17.

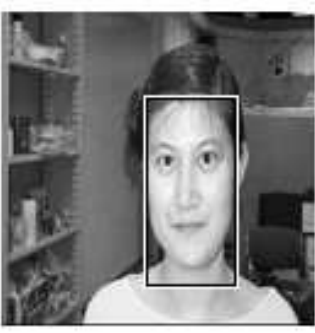

(c) PPL11.

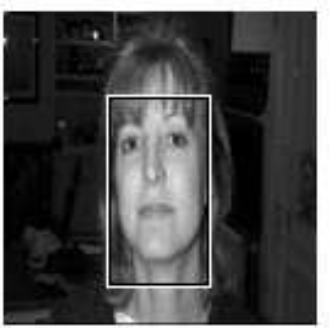

(f) PPL20.

Figure 3.1: Main subject detection algorithm's output.

of the pixels representing the main subject in the image matrix. After identifying the MICs and finding their location in the image matrix, the image matrix is reshaped into a vector, $\underline{x}$. This process simply concatenates the columns of the image matrix into a single vector. As a result, MICs will be located at different discontinuous locations in the reshaped image as exemplified in Figure 3.2. The location of these $M I C \mathrm{~s}$ is then tracked in vector $\underline{x}$ since this information is required when designing the UEP matrix, as will be discussed in Section 3.2.

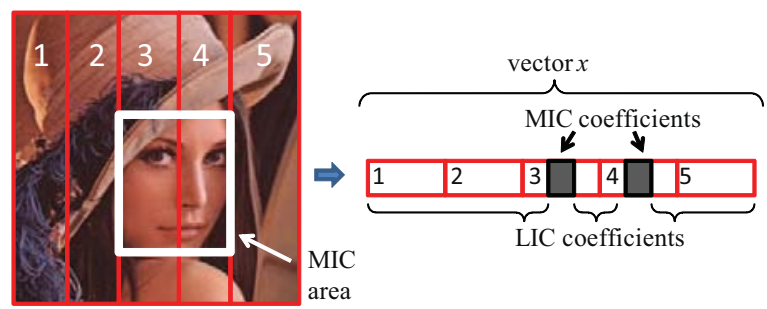

Figure 3.2: Main subject detection and distribution of its corresponding coefficients in $\underline{x}$. 


\subsection{Non-Uniform Protection for the Region of interest}

The region of interest in a given image is proposed to be recovered with a better quality by constructing the measurement matrix, $\phi$, in a special way as opposed to the completely random structure normally employed in CS. The ability to provide better quality to a region of interest is a very important functionality for image compression algorithms that it has been included in the JPEG 2000.

Sparse $\phi$ matrix is implemented in our algorithm as sparse $\phi$ matrices perform as good as dense ones and further provide less computational complexity for CS [14]. The performance comparison between dense and sparse $\phi$ matrices whose entries are taken from independent and identically distributed (i.i.d.) Gaussian distribution is shown in Figure 3.3. These matrices were chosen because they are incoherent with any basis of sparsity $\psi$ with high probability (universality property) [14].

Figure 3.3 shows the VIF of Lena images reconstructed with sparse and dense $\phi$ matrices when different number of measurements $(M)$ are taken. The number of measurements are chosen to represent $\operatorname{small}(M=2000, M=4000)$, medium $(M=6000)$ and large $(M=8000)$ number of measurements. From Figure 3.3, it could be seen that sparse and dense $\phi$ matrices have very similar performances at all number of measurements. The Lena images reconstructed using both the sparse and dense $\phi$ matrices are given in Figure 3.4 below for visual comparison.

Assume the total number of image coefficients in an image is $N$. Let $L \ll N$ be the number of non-zero entries in each row of $\Phi$, where $\Phi \in R^{m \times n}$. In equal error protection (EEP) setup, these $L$ non-zero elements are placed uniformly at random across the columns of each row and independent of other rows. Therefore, $\phi_{i, j}$ the element on the $i^{t h}$ row and $j^{\text {th }}$ column of $\Phi$ is an entry from iid gaussian distribution

or a zero with probabilities $\frac{L}{N}$ and $\frac{N-L}{N}$, respectively. This would clearly provide a uniform capturing of $\underline{x}$ coefficients by $M$ measurements $(\underline{y})$, as $\underline{y}=\Phi \underline{x}$.

In order to recover the region of interest (ROI) of a given image with a higher qual- 


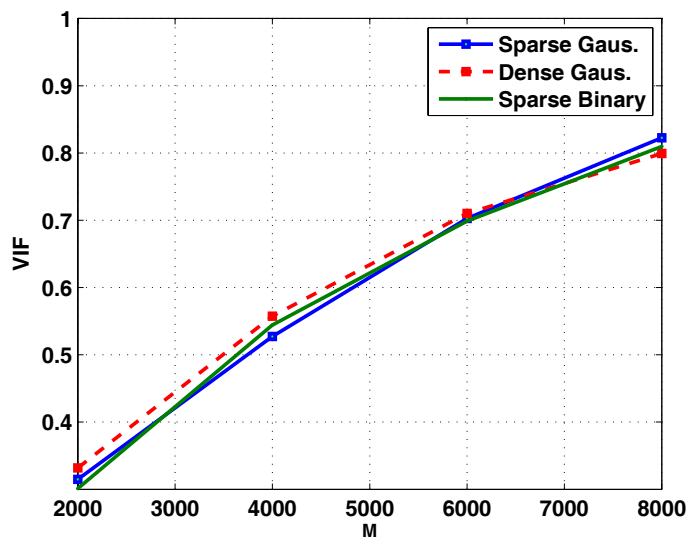

Figure 3.3: comparison of VIF for dense and sparse Gaussian matrices.

ity, more number of measurements have to be taken from coefficients that represent the ROI. This idea is inspired by the working principle of UEP erasure coding $[7,15]$. From the rule of matrix multiplication, it is clear that the $i^{\text {th }}$ image coefficient, $x_{i, 1}$ of vector $\underline{x}$, is multiplied by the entries in the $i^{\text {th }}$ column of the measurement matrix, $\phi$. By extension, a non-zero at $\phi_{j, i}$ takes a sample of the $i^{\text {th }}$ image coefficient, $x_{i, 1}$. Hence, we could take more measurements from the image coefficients representing the ROI by constructing the measurement matrix, $\phi$, in such a way that the columns of $\phi$ sampling the ROI coefficients are non-zeros with a higher probability than the rest of the image coefficients. As a result, the ROI will be recovered with a higher accuracy in CS reconstruction.

Therefore, for the sake of simplicity and without loss of generality two levels of importance $^{2}$ is assumed for the $N$ coefficients of $\underline{x}$ when designing the UEP property for the measurement matrix matrix, $\Phi$.

A sparse measurement matrix with fixed row weight, L, is proposed where each entry is non-zero with a probability $P_{1}$ or $P_{2}$ depending on its location in the matrix. Let $\alpha$ fraction of $N$ coefficients be more important coefficients (MICs) and $1-\alpha$

\footnotetext{
${ }^{2}$ We may also consider more than two levels of importance. However, this only increases the complexity of the problem while not improving the contribution.
} 

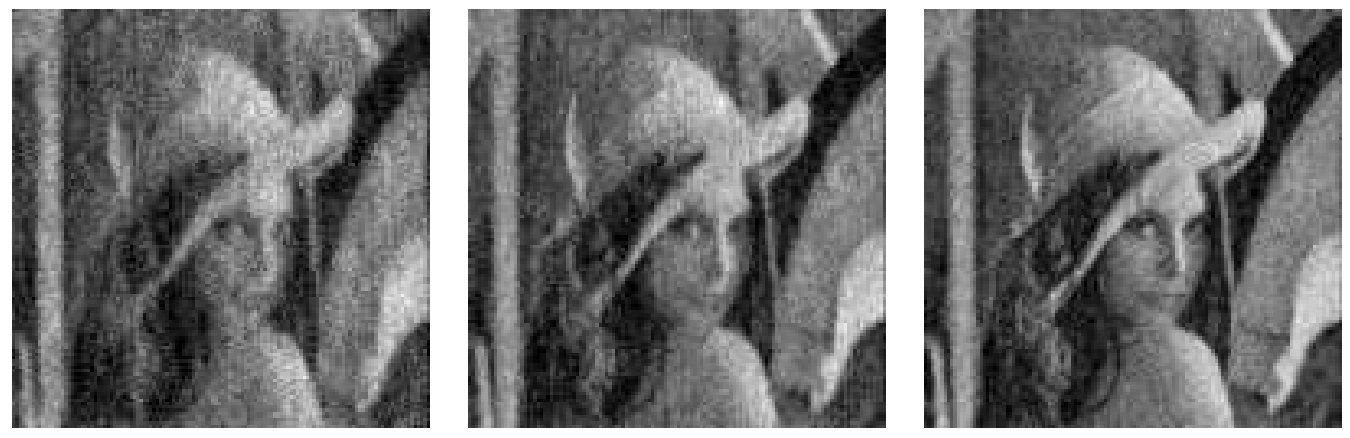

(a) Lena image reconstructed (b) Lena image reconstructed (c) Lena image reconstructed with dense $\Phi$ for $M=4000$. with dense $\Phi$ for $M=6000$. with dense $\Phi$ for $M=8000$.
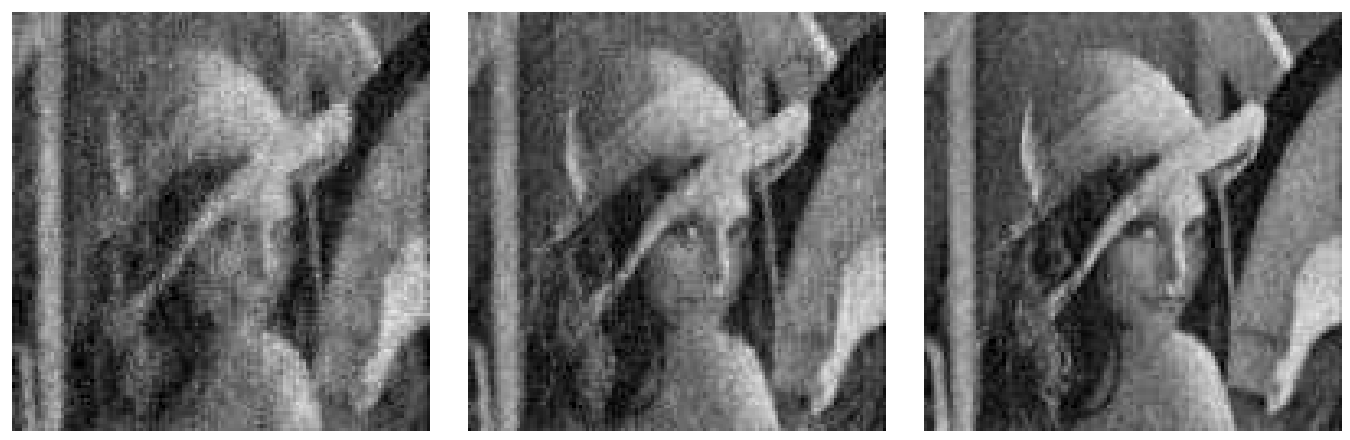

(d) Lena image reconstructed (e) Lena image reconstructed (f) Lena image reconstructed with sparse $\Phi$ for $M=4000$. with sparse $\Phi$ for $M=6000$. with sparse $\Phi$ for $M=8000$.

Figure 3.4: Visual performance comparison Lena images reconstructed with dense and sparse EEP- $\Phi$ s at different number of measurements.

fraction be less important coefficients (LICs). Clearly, the $n_{1}=\alpha N$ columns of $\Phi$ capture MIC and the rest $n_{2}=(1-\alpha) N$ capture LIC. Further, let us define $P_{1}=\frac{k_{M}}{N}$ and $P_{2}=\frac{k_{L}}{N}$, where $k_{M}$ and $k_{L}=\frac{1-\alpha k_{M}}{1-\alpha}$ are the protection levels. Clearly, UEP and EEP- $\Phi$ s are built by setting $k_{M}>k_{L}$ and $k_{M}=k_{L}=1$, respectively.

The encoding is then done as

$$
\underline{y}=\phi \underline{x}
$$

and the recovery is done as :

$$
\underline{\hat{s}}=\operatorname{argmin}\|s\|_{l_{1}} \text { s.t. } \underline{y}=\theta \underline{s}(\text { where } \theta=\phi \psi) .
$$


The estimated image, $\underline{\hat{X}}$, is finally obtained as:

$$
\underline{\hat{X}}=\psi \underline{\hat{s}}
$$

A regular basis pursuit (BP) reconstruction algorithm [12] (which is designed employing linear programming techniques) is then employed to solve the CS reconstruction problem (2.1). The quality of reconstructed images is finally assessed using visual information fidelity (VIF) [16]. This image quality assessment is chosen as it is able to measure image quality that relates with visual perception. Note that VIF of a reconstructed image varies between 0 and 1 , where a higher value means a better reconstruction quality.

\subsection{Optimal Parameter values}

Several experiments were carried out using the sample images, shown in Figure 3.1, in order to find the optimal value for the row weight, $L$, of $\phi$. Figure 3.5 shows the reconstruction quality of Lena image at different $L$ and $k_{M}$ values when $M=$ $8192, \alpha \approx 0.15$ and implementing the DCT as the basis of sparsity, $\psi$.

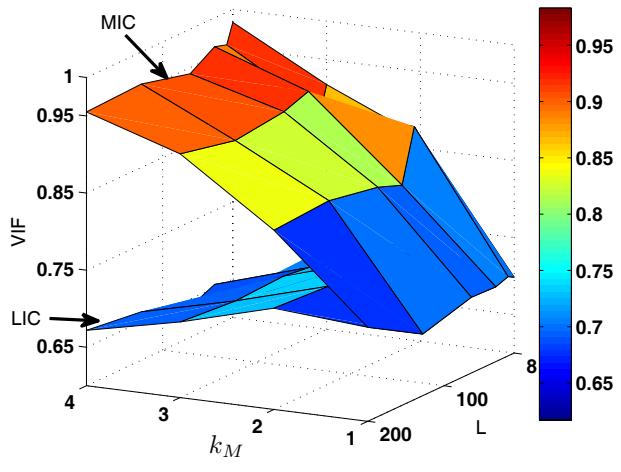

(a) $\mathrm{m}=6000$

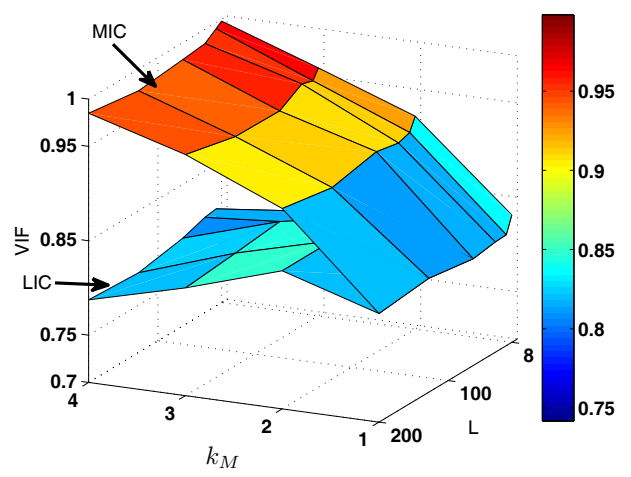

(b) $\mathrm{m}=8192$

Figure 3.5: VIF of reconstructed Lena image at $\mathrm{m}=8192$, for different $k_{M}$ and $L$ values 
As it could be seen from Figure 3.5, different values of $L$ result in similar reconstruction quality for all $k_{M}$ values. However, the CPU time is significantly higher when a large value of $L$ is used. It could also be seen, from Figure 3.5, that the quality of the $M I C$ s increases while that of the $L I C$ s decreases when increasing the $k_{M}$ values. However, the performance of neither the $M I C$ s nor the $L I C$ s show significant change with various $L$ values. Similar results were obtained for all the images experimented with, for different number of measurements $M$ and also when the wavelet basis is used as the basis of sparsity, $\psi$. A row weight of $16, L=16$, is thus chosen to be the optimal row weight in order to achieve fast encoding/decoding process.

Another parameter that is important for UEP on CS is the size of the image that is selected to be the region of interest (ROI) as compared to the size of the whole image, which is represented by $\alpha$. Figure 3.6 below shows the reconstruction quality of Lena image at different $\alpha$ and $k_{M}$ values when $M=8192, L=16$ and implementing the DCT as the basis of sparsity, $\psi$.

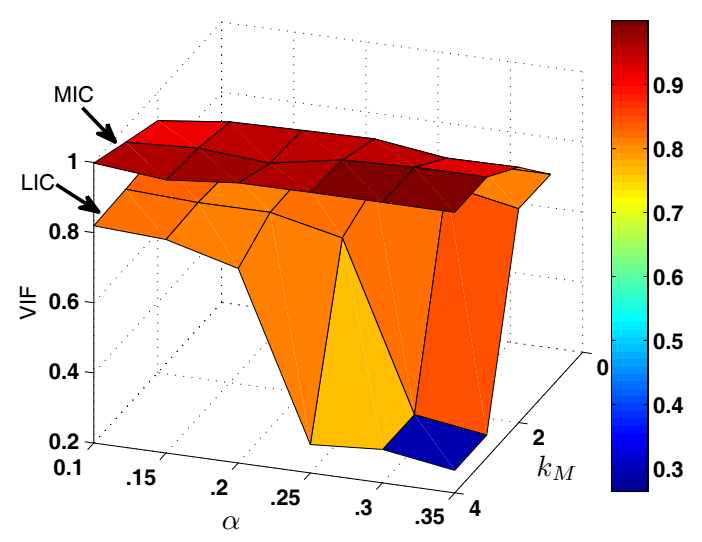

Figure 3.6: VIF of reconstructed Lena image at $\mathrm{M}=8192$ and $\mathrm{L}=16$ for different $\alpha$ and $k_{M}$ values.

As it could be seen from Figure 3.6, a small size of ROI could be reconstructed in such a way that the ROI is recovered with a quality better than the background whereas the background is also reconstructed with an acceptable quality. Where 
as, the quality of the background significantly drops to an unacceptable level when large ROIs are used. This is because, as the size of the ROI is large, most non-zeros would be allocated for columns of $\phi$ measuring the ROI while the rest columns have insufficient non-zero entries to successfully sample the background. Note that the MICs represent the ROI while the LICs represent the background.

Another interesting relationship could be observed between $k_{M}$ values and $M I C$ sizes as shown in Figure 3.6. It could be seen that it is possible to reconstruct large ROIs with a quality better the background while the background is also reconstructed with an acceptable quality by using small $k_{M}$ values. In Figure 3.6, for example, the quality of the LICs is very low for $\alpha=0.25$ when $k_{M}=4$. However, it could be seen that the LICs are reconstructed with a significantly better quality when the $k_{M}$ value is decreased to $k_{M}=3$. The trade off is that when a smaller $k_{M}$ value is used, the difference between the quality of MICs and LICs decreases with $k_{M}=1$ being EEP where the ROI and the background have similar qualities.

As it could be seen in Figure 3.6, the maximum MIC size that results in acceptable recovery of LICs for a $k_{M}$ value of 4 is obtained when $\alpha \approx 0.2$ (i.e. when the ROI covers $20 \%$ of the whole image coefficients). Similar result was obtained for a $k_{M}$ value of 5. The largest of the ROIs for the sample images shown in Figure 3.1 approximately covers $20 \%$ of the whole image $(\alpha \approx 0.2)$. The size of the ROIs was purposely made to be of this small size by adopting a slight modification in the MSD algorithm[13]. Moreover, a $k_{M}$ value of $k_{M}=5$ is used as it is found to provide good reconstruction for both the ROI and background when $\alpha \leq 0.2$.

Some of the Lena images that correspond to the plot in Figure 3.6 are shown in Figure 3.7 for Visual performance comparison.

Figure 3.7 shows the EEP $\left(k_{M}=1\right.$ ) and UEP (for $k_{M}=3$ and $k_{M}=4$ ) reconstruction of Lena images when different sizes of region of interest (ROI), and hence different $\alpha$ values, are used. Lena images shown in Figures 3.7(a), 3.7(d), 3.7(g) and 


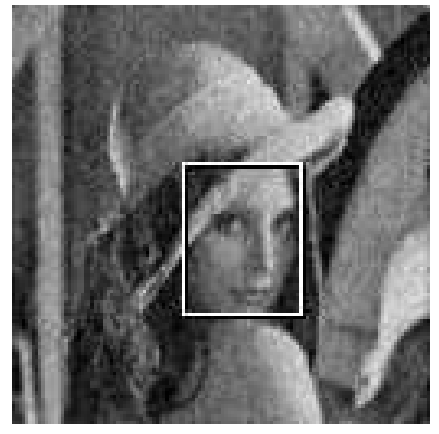

(a) EEP, $\alpha=0.1$

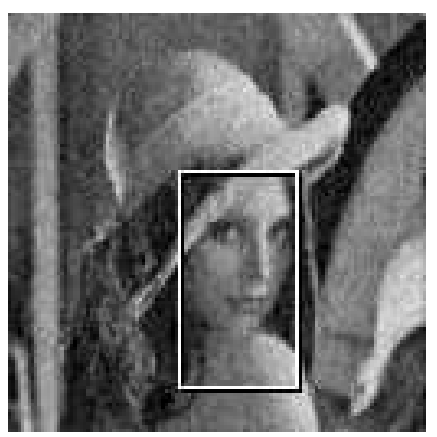

(d) EEP, $\alpha=0.15$

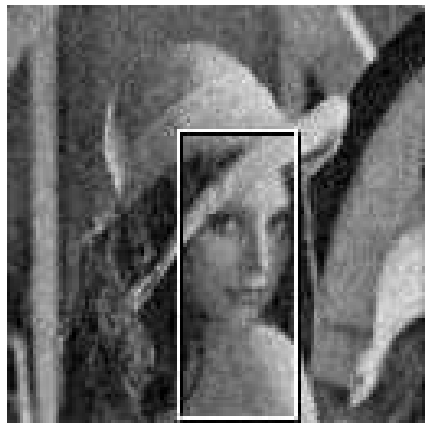

(g) EEP, $\alpha=0.2$

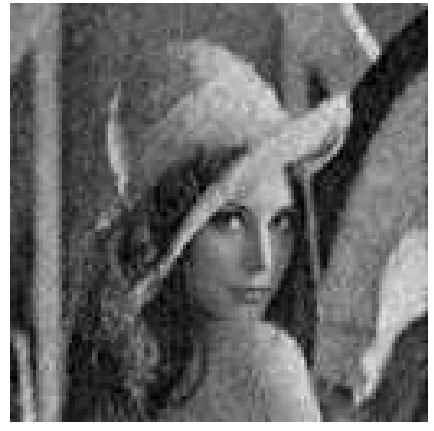

(b) $U E P, k_{M}=3$

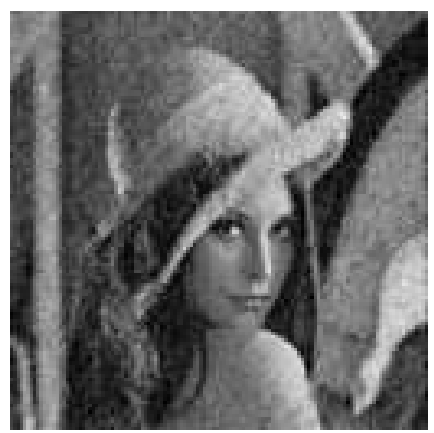

(e) $U E P, k_{M}=3$

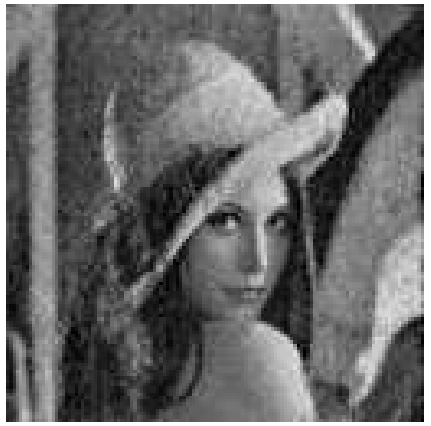

(h) $U E P, k_{M}=3$

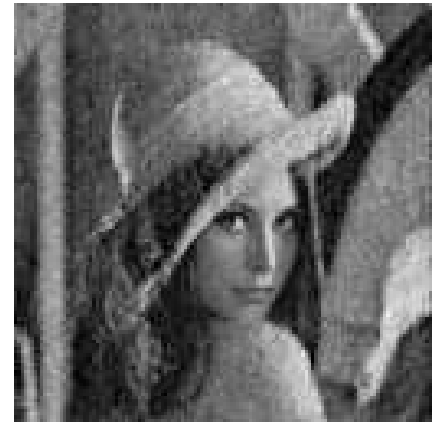

(c) $U E P, k_{M}=4$

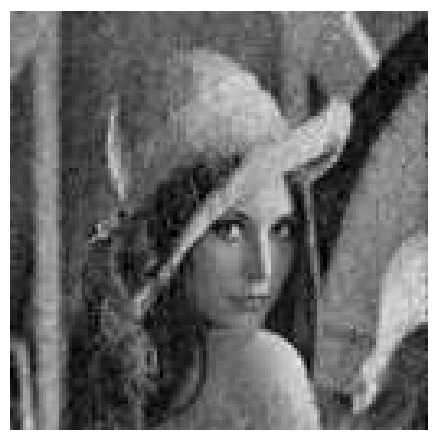

(f) $U E P, k_{M}=4$

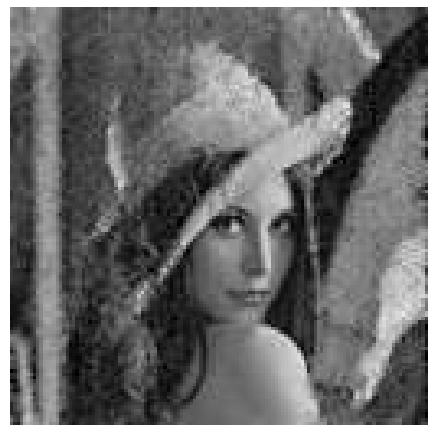

(i) $U E P, k_{M}=4$

3.7(j) are reconstructed using EEP- $\Phi$. The rectangles in these figures show the part of the image selected to be the regions of interest(ROIs) for the UEP application. The values of $\alpha$ are $\alpha \approx 0.1, \alpha \approx 0.15, \alpha \approx 0.2$ and $\alpha \approx 0.25$ respectively for ROIs shown in Figures 3.7(a), 3.7(d), 3.7(g) and 3.7(j). 


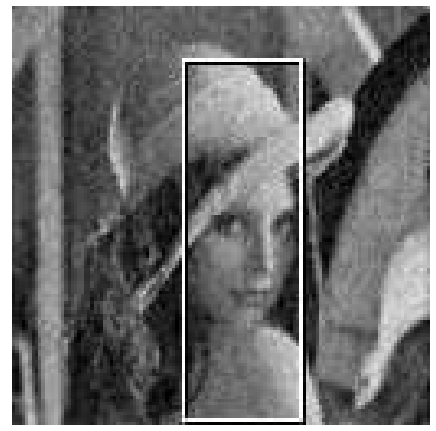

(j) EEP, $\alpha=0.25$

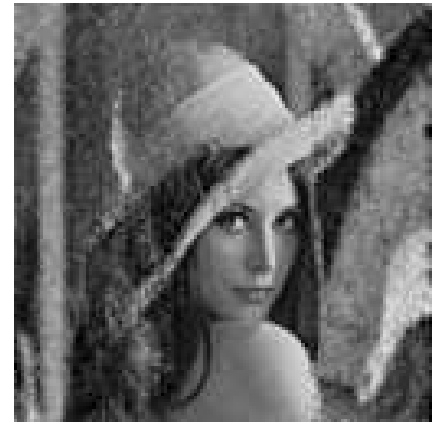

(k) $U E P, k_{M}=3$

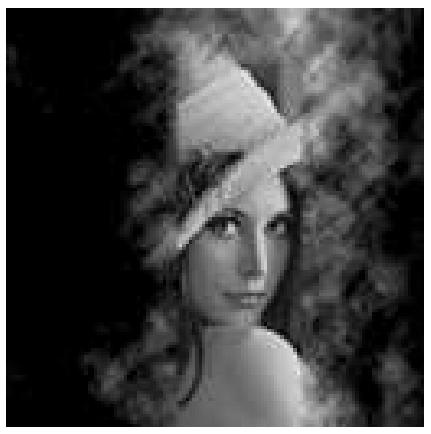

(l) $U E P, k_{M}=4$

Figure 3.7: Visual performance comparison of Lena image for different $\alpha$ and $k_{M}$ values.

From Figure 3.7, we could see that the ROIs have been reconstructed with a much better quality by using the proposed UEP- $\phi$ when compared with the EEP$\phi$. Moreover, the ROI is reconstructed with a better quality for higher $k_{M}$ values. It could also be seen that, when the portion of the image selected as the region of interest (ROI) is not very large, the background is reconstructed with a quality very close to the EEP. However, if the ROI is very large the background could be significantly degraded for large $k_{M}$ values. In Figure 3.7 for example, the background is significantly degraded when $k_{M}=4$ when $\alpha \approx 0.25$ (Figure 3.7(l)). This could be avoided by using a smaller $k_{M}$ value, for example $k_{M}=3$ provides very good results when $\alpha \approx 0.25$ as shown in Figure 3.7(k).

\subsection{Relationship between parameters and image quality}

In this section, we discuss how the reconstructed image quality varies with the number of measurements, $M$, and the protection level $k_{M}$. In Section 3.3, it has been mentioned that results similar to those shown in Figures 3.5 and 3.6 are obtained for different number of measurements, $M$. From the theory of CS, it is known that the reconstruction error decreases when the number of measurements, $M$, is increased. 
As a result, it is clear that even though similar pattern as that of Figures 3.5 and 3.6 is obtained with different $M$ values, the actual VIF values increase when $M$ is large and decrease when $M$ is small.

Figure 3.8 shows the reconstruction quality of the Lena image at different number of measurements, $M$. The results are presented for $\operatorname{EEP}\left(k_{M}=1\right)$ and $\operatorname{UEP}\left(k_{M}=4\right)$. The row weight is set to a value of $16, L=16$, in both cases. A very important observation that could be made from Figure 3.8 is the successfulness of UEP. More precisely, it could be seen that the quality of the ROI (VIF of MICs) is significantly better than the background (VIF of LICs). Moreover, the background is recovered with a quality that is as good as the EEP.

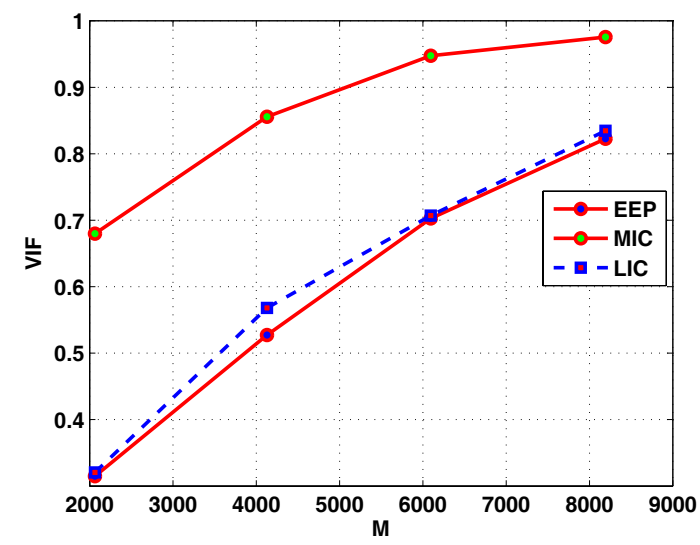

Figure 3.8: Reconstruction quality of Lena image for $k_{M}=4, \mathrm{~L}=16$ and $\alpha=0.15$ at different $M$ values.

Figure 3.9 further emphasizes the relationship between the VIF of ROIs (MICs) and background (LICs) which could also be observed in Figure 3.6. The result shown in Figure3.9 is for the Lena image when $M=8192, \alpha \approx 0.15$ and $L=16$. It could be seen from this figure that the quality of the ROI increases when high $k_{M}$ values are used where as the background is recovered with a quality as good as that of $\operatorname{EEP}\left(k_{M}=1\right)$. It should however be noted that the size of the ROI must be appropriate according to the the discussion in Section 3.3 for this relationship to 


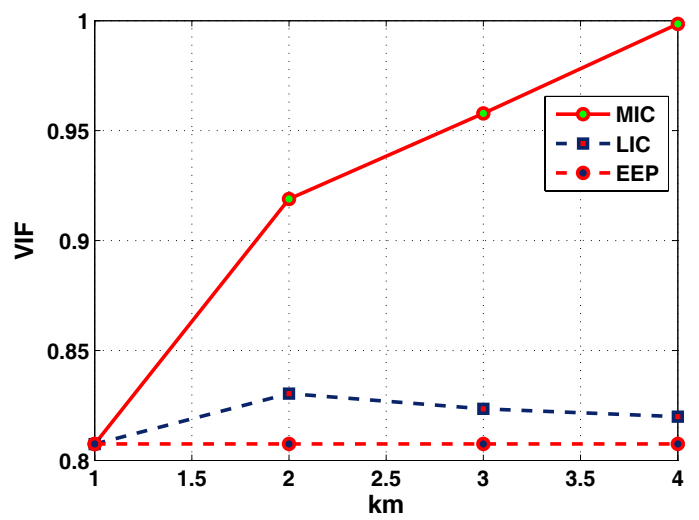

Figure 3.9: Reconstruction quality of Lena image for $\mathrm{M}=8192$ and $\alpha=0.15$ at different $k_{M}$ values.

hold.

\subsection{Simulation results on sample images}

In this section, the simulation results obtained when implementing the EEP- and UEP- $\phi$ s on the images shown in Figure 3.1 are presented. We remind that the proposed UEP measurement matrix, designed as discussed in Section 3.2, is used at the encoding stage $(\underline{y}=\Phi \underline{x})$. The recovery process is then carried out using a regular basis pursuit $(\mathrm{BP})$ reconstruction algorithm [12].

All images are first resized to a size of $128 \times 128$, resulting a total number of 16,384 coefficients $(N=16,384)$. Then, the optimal parameter values (presented in Section 3.3) are used to obtain all the simulation results presented in this section. The number of measurement used for results presented in Table 3.1 is $M=6000$. A row weight value of $L=16$ is used for all simulations while a $k_{M}$ value of $\mathrm{k}_{M}=5$ is used for all the images except for sample images PPL12 and PPL20. A $k_{M}$ value of $\mathrm{k}_{M}=4$ is used for PPL12 and PPL20 due to their large $\alpha$ values (please refer Table 3.1 for the $\alpha$ s of the images).

In Table 3.1, we compare the VIF of MIC area, LIC area, and the whole image for 
UEP- $\Phi$ encoding versus encoding with sparse and dense EEP- $\Phi$ s. The VIF for EEP is computed for the whole image. On the other hand, the VIF for UEP is computed for the image as a whole and also for the MICs and LICs separately. The VIF computed for the part of an image identified as the ROI, which is shown under a rectangle in Figure 3.1, is shown as the VIF for the MIC. In a similar way, the VIF computed for the whole image excluding the main subject is shown as the VIF for the LIC.

Table 3.1: VIF of CS reconstruction performance employing EEP- $\Phi$ and UEP- $\Phi$.

\begin{tabular}{|c|r|r|r|r|r|r|r|r|}
\hline \multicolumn{2}{|c|}{ Images } & P2 & P10 & P11 & P12 & P17 & P20 \\
\hline \multirow{7}{*}{ VIF } & \multirow{2}{*}{ EEP } & Sparse & 0.83 & 0.67 & 0.75 & 0.69 & 0.81 & 0.8 \\
\cline { 3 - 8 } & & Dense & 0.85 & 0.64 & 0.78 & 0.71 & 0.82 & 0.77 \\
\cline { 2 - 8 } & \multirow{2}{*}{ UEP } & MIC & 1 & 0.98 & 0.99 & 0.96 & 1 & 0.97 \\
\cline { 3 - 8 } & LIC & 0.81 & 0.64 & 0.74 & 0.69 & 0.84 & 0.76 \\
\cline { 3 - 8 } & Total & 0.87 & 0.68 & 0.77 & 0.71 & 0.84 & 0.85 \\
\hline
\end{tabular}

From Table 3.1, we can see that when UEP- $\Phi$ is employed in the encoding phase a considerable improvement is obtained for the region of interest (ROI) at the expense of a small quality loss in the background. For instance, for the image PPL11, the VIF of MIC has increased from 0.77 (for dense EEP- $\Phi$ ) to 0.99 (an almost perfect reconstruction), while the VIF of LIC has a small decrease from 0.77 to 0.74 . Therefore, the deterioration in the background may not even be noticed even though the region of interest (ROI) has been reconstructed with a quality very close to the original. This could be clearly seen in Figure 3.12.

Finally, we present the images reconstructed from UEP-, sparse EEP- and dense EEP- $\phi$ s in Figures 3.10 to 3.15 for visual comparisons. Note that the DCT has been used as the basis of sparsity for the simulations presented in this chapter. Results obtained when implementing the wavelet basis are presented in Appendix A.

From Figures 3.10 to 3.15, we can see that the region of interest (ROI) has been 


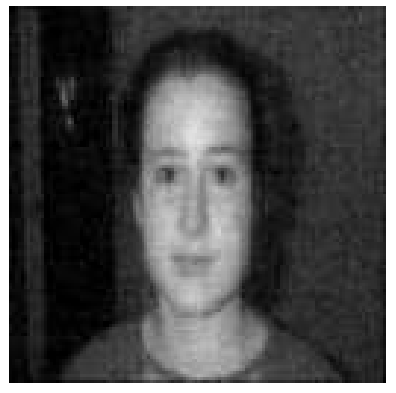

(a) Sparse EEP- $\Phi$

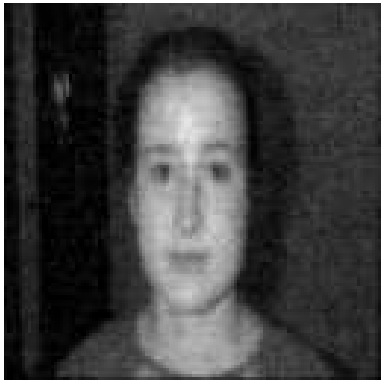

(b) Dense EEP- $\Phi$

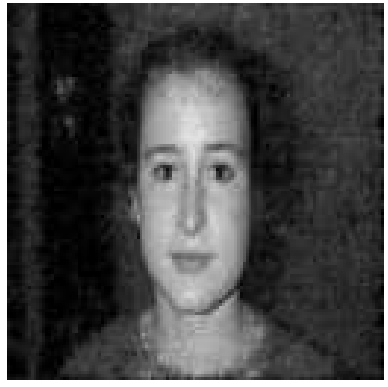

(c) UEP- $\Phi$

Figure 3.10: Reconstruction quality comparison of UEP- $\phi$ versus dense and sparse EEP- $\phi$ s for sample image PPL2. Here, $n=16384$ and $m=6000$.

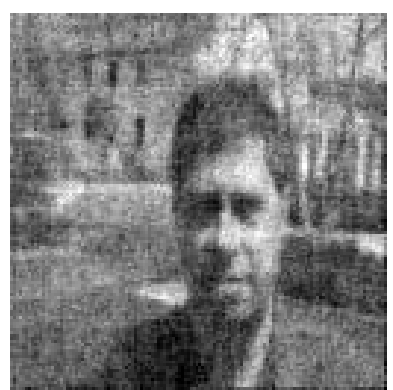

(a) Sparse EEP- $\Phi$

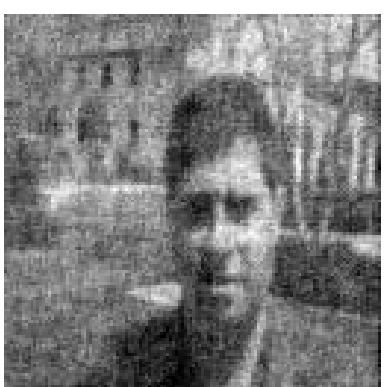

(b) Dense EEP- $\Phi$

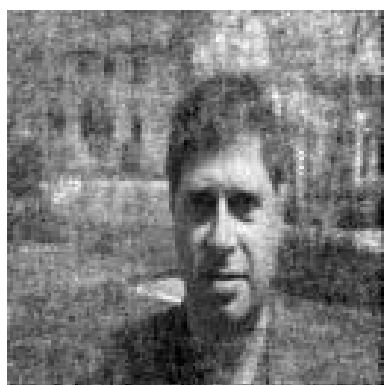

(c) UEP- $\Phi$

Figure 3.11: Reconstruction quality comparison of UEP- $\phi$ versus dense and sparse EEP- $\phi$ s for sample image PPL10. Here, $n=16384$ and $m=6000$.

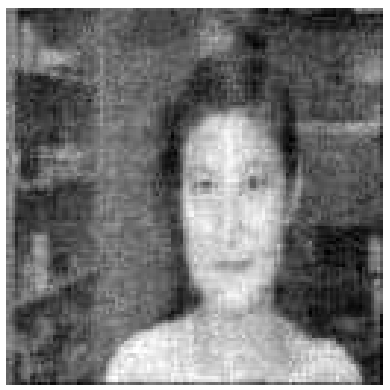

(a) Sparse EEP- $\Phi$

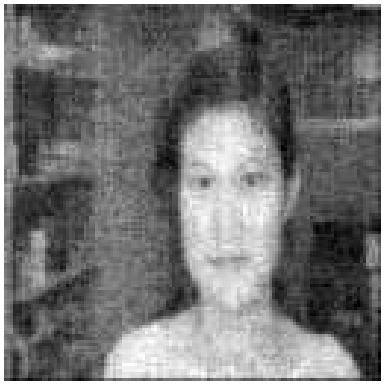

(b) Dense EEP- $\Phi$

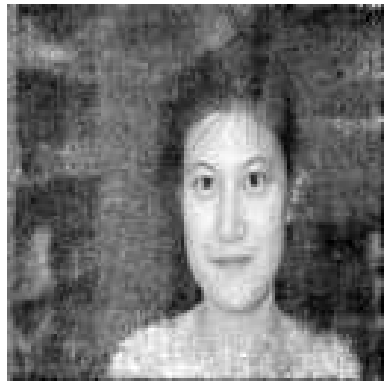

(c) UEP- $\Phi$

Figure 3.12: Reconstruction quality comparison of UEP- $\phi$ versus dense and sparse EEP- $\phi$ s for sample image PPL11. Here, $n=16384$ and $m=6000$. 


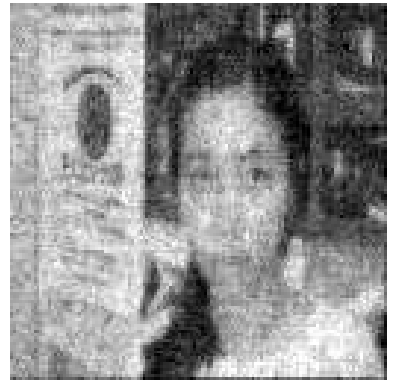

(a) Sparse EEP- $\Phi$

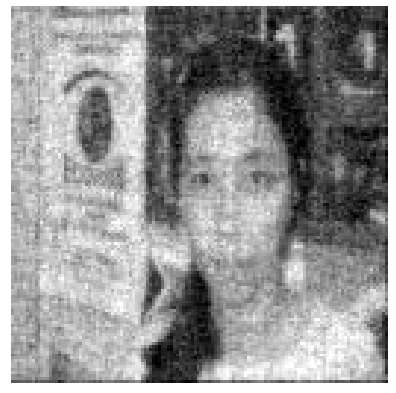

(b) Dense EEP- $\Phi$

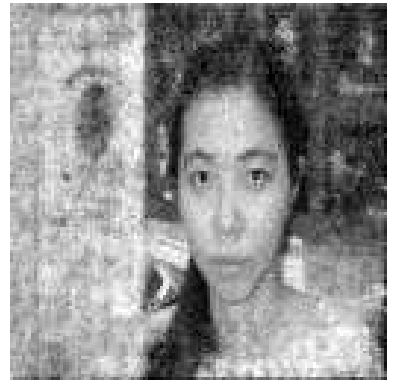

(c) UEP- $\Phi$

Figure 3.13: Reconstruction quality comparison of UEP- $\phi$ versus dense and sparse EEP- $\phi$ s for sample image PPL12. Here, $n=16384$ and $m=6000$.

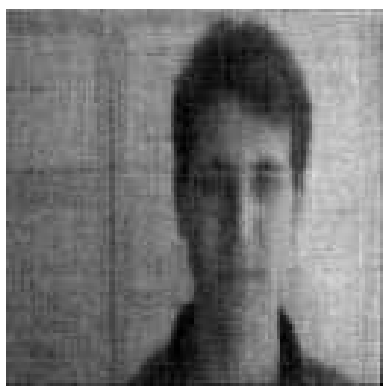

(a) Sparse EEP- $\Phi$

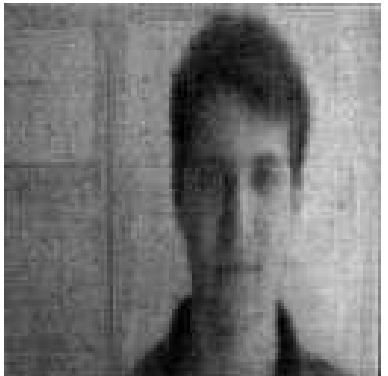

(b) Dense EEP- $\Phi$

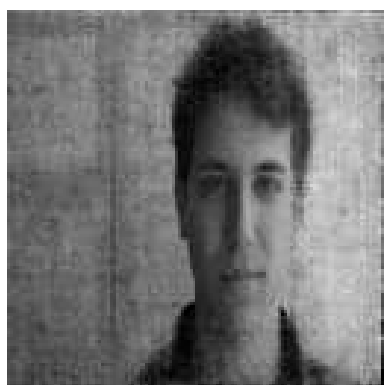

(c) UEP- $\Phi$

Figure 3.14: Reconstruction quality comparison of UEP- $\phi$ versus dense and sparse EEP- $\phi$ s for sample image PPL17. Here, $n=16384$ and $m=6000$.

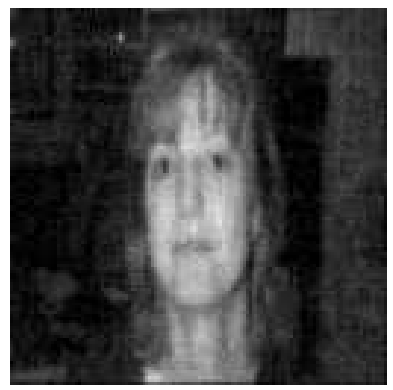

(a) Sparse EEP- $\Phi$

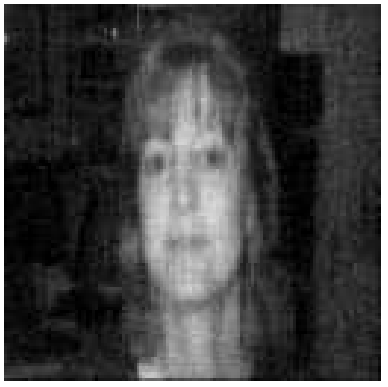

(b) Dense EEP- $\Phi$

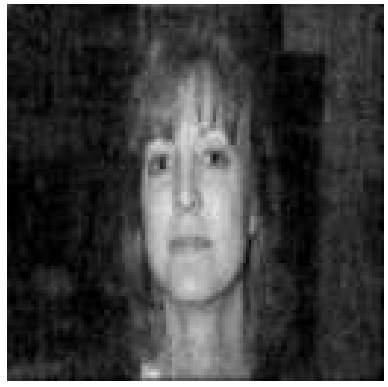

(c) UEP- $\Phi$

Figure 3.15: Reconstruction quality comparison of UEP- $\phi$ versus dense and sparse EEP- $\phi$ s for sample image PPL20. Here, $n=16384$ and $m=6000$. 
recovered with a better quality than the rest of image for all the images without significant loss of quality in the background. Therefore, we have been able to better protect the region of interest of images by employing our proposed structure of UEP$\Phi$. 


\section{CHAPTER 4}

\section{Providing more protection for images in transform basis}

\subsection{Providing more protection for the whole image}

In this section, an algorithm is proposed which exploits the prior knowledge about the structure of transform image coefficients so as to achieve a better recovery quality for the whole image.

\subsubsection{The structure of transform image coefficients}

As it has been discussed in Section 2.2, images have a concise representation in some transform basis such as DCT and wavelet. The representation of images in these transform bases has a certain common structure. This is because, the standard methods of finding the DCT and wavelet transforms also include a process of sorting the obtained transform coefficients to provide a certain structure for the output.

Consider an image coefficients vector $\underline{x}$ and its sparse representation $\underline{s}$. As mentioned in Section 2.2, high variations in the neighboring pixels cannot be seen in the spatial representation of images due to the high correlation between these neighboring pixels.

However, when the frequency components of a natural image is extracted employing the DCT transform, most coefficients of $\underline{s}$ are close to zero for high frequencies. Therefore, the information of the image is confined within a few coefficients representing low frequencies. DCT further separates frequencies and places the low frequencies at the beginning of the transform coefficients vector, $\underline{s}$.

Similarly, when an image is projected onto the wavelet basis, the coefficients placed 
at the beginning of $\underline{s}$ correspond to the parent coefficients (the root of the wavelet trees) [5]. Therefore, in wavelet domain as well as DCT domain the large transform coefficients are concentrated at the beginning of the $\underline{s}$.

Figures 4.1 and 4.2 below show the output of wavede 2 command in Matlab, which finds the two-dimensional wavelet decomposition of an image, and how it functions. An image of size $512 \times 512$ is assumed to undergo an $n$-level decomposition in Figure 4.1 .

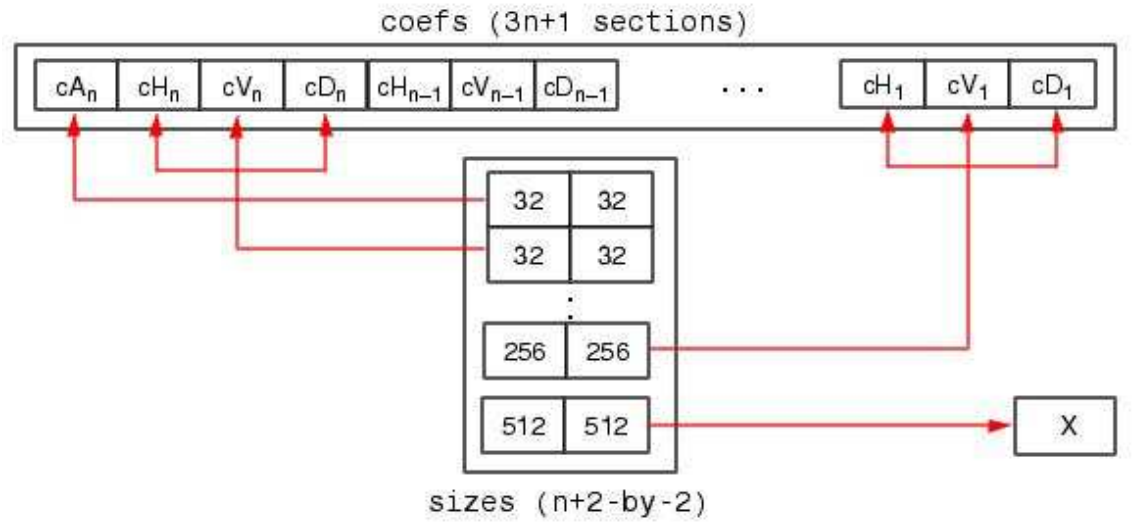

Figure 4.1: Two-dimensional wavelet decomposition in Matlab

The sparse transform coefficients vector, $\underline{s}$, will be constructed as: $\underline{\mathrm{s}}=[\mathrm{A}(\mathrm{n}) \mid$ $\mathrm{H}(\mathrm{n})|\mathrm{V}(\mathrm{n})| \mathrm{D}(\mathrm{n})|\ldots \mathrm{H}(\mathrm{n}-1)| \mathrm{V}(\mathrm{n}-1)|\mathrm{D}(\mathrm{n}-1)| \ldots|\mathrm{H}(1)| \mathrm{V}(1) \mid \mathrm{D}(1)]$.

where $\mathrm{n}$ is the level of decomposition and A stands for the approximation coefficients vector while the $\mathrm{H}, \mathrm{V}$ and $\mathrm{D}$ stand for the horizontal, vertical and diagonal detail coefficients respectively.

This two-dimensional DWT basically involves filtering and down sampling the image coefficients as shown in Figure 4.2. In this decomposition, sparse coefficients that correspond to the high activity area of the image are represented by the subbands HL, LH and HH while the transform coefficients that correspond to the low 
activity area of the image are represented by the LL sub-band. These HL, LH, HH and LL sub-bands correspond to the H, V, D and A of Figure 4.2 respectively.

Figure 4.2 shows the decomposition process carried out at each decomposition level. When $n$-level of decomposition is required this process is repeated $n$ times. Obviously, the input for the first level decomposition is the image while the input for the next levels of decomposition is the out put given by the LL sub-band from the previous decomposition level.

Human eye is normally attracted to the low activity area of the image and hence sensitive to changes in these areas. From Figures 4.1 and 4.2 these areas are evidently represented by transform coefficients located at the beginning of $\underline{s}$. Further more, as discussed in Section 2.2, these areas are characterized by having significant values in the frequency domain.

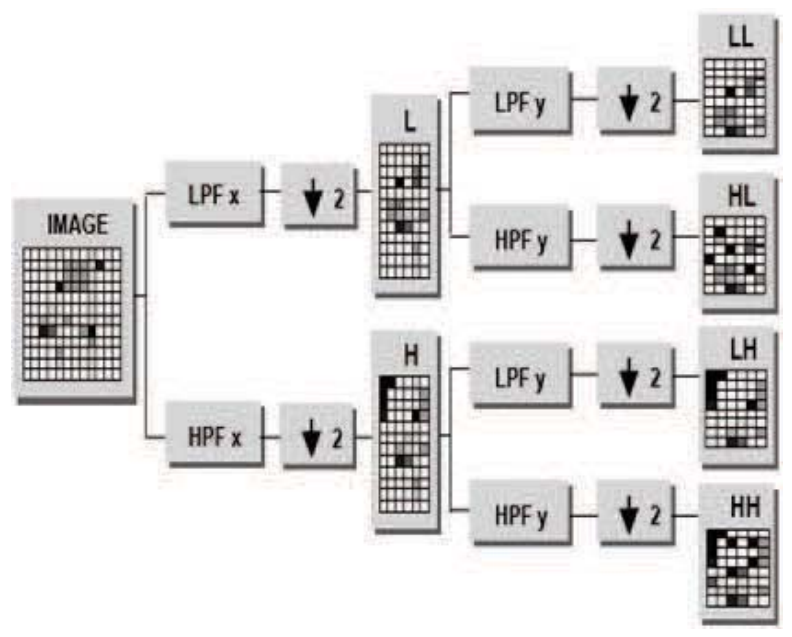

Figure 4.2: The decomposition process of the two-dimensional DWT. LPF and HPF stand for Low-Pass Filter and High-Pass Filter respectively.

\subsubsection{Non-uniform sampling of transform image coefficients}

Assume an image has been already transformed to DCT or wavelet transform domains and $\underline{s}=\Psi^{-1} \underline{x}=\Psi^{T} \underline{x}$ has been obtained. In contrast to our algorithm in Section 3.2, 
the sampling is performed by $\underline{y}=\Phi \underline{s}$ instead of $\underline{y}=\Phi \underline{x}$. We construct a UEP- $\Phi$ to protect the more important transform coefficients with a similar setup described in Section 3.2. This construction procedure for the proposed $\Phi$ is discussed again below for the sake of completeness.

Let $L \ll N$ be the number of non-zero entries in each row of $\Phi$. In the EEP setup, these $L$ non-zero elements are placed uniformly at random across the columns of each row and independent of other rows. Therefore, $\phi_{i, j}$ the element on the $i^{\text {th }}$ row and $j^{\text {th }}$ column of $\Phi$ is an entry from iid gaussian distribution or a zero with probabilities $\frac{L}{N}$ and $\frac{N-L}{N}$, respectively.

However, as we discussed in the previous section, we have the knowledge that significant (important) coefficients are concentrated at the beginning of $\underline{s}$. Consequently, employing the idea of UEP erasure coding from $[7,15]$, we propose to concentrate the $L$ non-zero elements of $\Phi$ toward the beginning columns of $\Phi$. This is because, more important coefficients are incorporated in the generation of more measurements with this setup. As a result, these coefficients will be recovered with a higher accuracy in CS reconstruction. Consequently, a better reconstruction quality is achieved for the whole image as opposed to the quality improvement provided particularly for the ROI as proposed in the previous section (Section 3.2).

Therefore, for the sake of simplicity and without loss of generality we assume that $N$ coefficients of $\underline{s}$ are grouped into two levels of importance. Let $\alpha$ fraction of $N$ coefficients be more important coefficients (MIC) and $1-\alpha$ fraction be less important coefficients (LIC). Clearly, the first $n_{1}=\alpha N$ columns of $\Phi$ capture MIC and the rest $n_{2}=(1-\alpha) N$ capture LIC.

Let $P_{1}$ and $P_{2}$ be the probabilities that an element in the first $n_{1}$ or last $n_{2}$ columns of a particular row of $\Phi$ be non-zero, respectively. Further, let us define $P_{1}=\frac{k_{M}}{N}$ and $P_{2}=\frac{k_{L}}{N}$, where $k_{M}$ and $k_{L}=\frac{1-\alpha k_{M}}{1-\alpha}$ are the protection levels. Clearly, UEP and EEP- $\Phi$ s are built by setting $k_{M}>k_{L}$ and $k_{M}=k_{L}=1$, respectively. In the next 
section, we implement our proposed algorithm and evaluate its performance.

\subsubsection{Non-Uniform Sampling Implementation on Sample Images}

First, without loss of generality we employ the wavelet transform to obtain the sparse representation of images. In addition, we assume a regular basis pursuit (BP) reconstruction algorithm [12] is employed to solve the CS reconstruction problems in this section. We find the optimal values of $\alpha, L$ and $k_{M}$ for various values of $M$ as in

Section 3.3. We found that for $N=16384$ the optimal value of $\alpha, L$, and $k_{M}$ are approximately $\alpha \approx 0.25, L=100$, and $k_{M}=5$. This means that the $25 \%$ beginning coefficients of $\underline{s}$ should be more protected than the rest.

We perform our simulations on the sample images (shown in Figure 3.1) with these optimal parameters. Note that all the sample images have a size of $128 \times 128$. We, then, compare the VIF of images reconstructed by employing our proposed algorithm (UEP- $\phi$ for whole image, Section 4.1), EEP- $\Phi$ and TSWCS [5] in Table 4.1 for various $M$ values.

From Table 4.1, we can observe a considerable improvement in the quality of the reconstructed images when UEP- $\Phi$ has been employed. We can observe that the quality of images reconstructed by employing UEP- $\phi$ for whole image with $M=4000$ is equivalent to the quality of the images reconstructed by employing EEP- $\Phi$ or TSWCS with $M=8000$. We also visually compare the performance of EEP and TSWCS with UEP- $\phi$ for whole image using the sample images in Figures 4.3 to 4.8 when $M=4000$.

From Figures 4.3 to 4.8 we can see that the quality of the reconstructed images is significantly better when $U E P-\phi$ for whole image is employed. We can also see that the quality of images reconstructed using the TSWCS is better than that obtained by employing an EEP- $\Phi$. However, TSWCS does not result in a reconstruction quality as good as that achieved by employing UEP- $\phi$ for whole image. 
Table 4.1: VIF measure of the reconstructed images employing our proposed algorithm versus TSWCS and encoding with EEP- $\Phi$.

\begin{tabular}{|c|c|c|c|c|c|c|}
\hline Image & PPL2 & PPL10 & PPL11 & PPL12 & PPL17 & PPL20 \\
\hline \multicolumn{7}{|c|}{$M=2000$} \\
\hline EEP- $\Phi$ & 0.489 & 0.169 & 0.362 & 0.304 & 0.414 & 0.473 \\
\hline TSWCS & 0.821 & 0.491 & 0.681 & 0.539 & 0.697 & 0.680 \\
\hline UEP- $\Phi$ & 0.825 & 0.546 & 0.655 & 0.621 & 0.736 & 0.726 \\
\hline \multicolumn{7}{|c|}{$M=4000$} \\
\hline EEP- $\Phi$ & 0.765 & 0.466 & 0.640 & 0.508 & 0.67 & 0.672 \\
\hline TSWCS & 0.908 & 0.657 & 0.802 & 0.714 & 0.836 & 0.820 \\
\hline UEP- $\Phi$ & 0.978 & 0.924 & 0.959 & 0.934 & 0.967 & 0.958 \\
\hline \multicolumn{7}{|c|}{$M=6000$} \\
\hline EEP- $\Phi$ & 0.879 & 0.634 & 0.737 & 0.713 & 0.805 & 0.805 \\
\hline TSWCS & 0.955 & 0.771 & 0.876 & 0.818 & 0.914 & 0.901 \\
\hline UEP- $\Phi$ & 0.980 & 0.932 & 0.963 & 0.941 & 0.968 & 0.962 \\
\hline \multicolumn{7}{|c|}{$M=8000$} \\
\hline EEP- $\Phi$ & 0.929 & 0.768 & 0.852 & 0.793 & 0.891 & 0.873 \\
\hline TSWCS & 0.984 & 0.835 & 0.921 & 0.876 & 0.957 & 0.934 \\
\hline UEP- $\Phi$ & 0.980 & 0.932 & 0.963 & 0.941 & 0.968 & 0.962 \\
\hline
\end{tabular}

As we discussed earlier, our proposed algorithms are implemented at the encoding phase only by altering the $\Phi$ construction. Therefore, our proposed algorithm can even be combined with CS reconstruction algorithms that propose novel decoding based on the known structure of images such as TSWCS [5]. We perform simulations to see if the combination of our proposed algorithm with TSWCS, referred to as TSWCS 

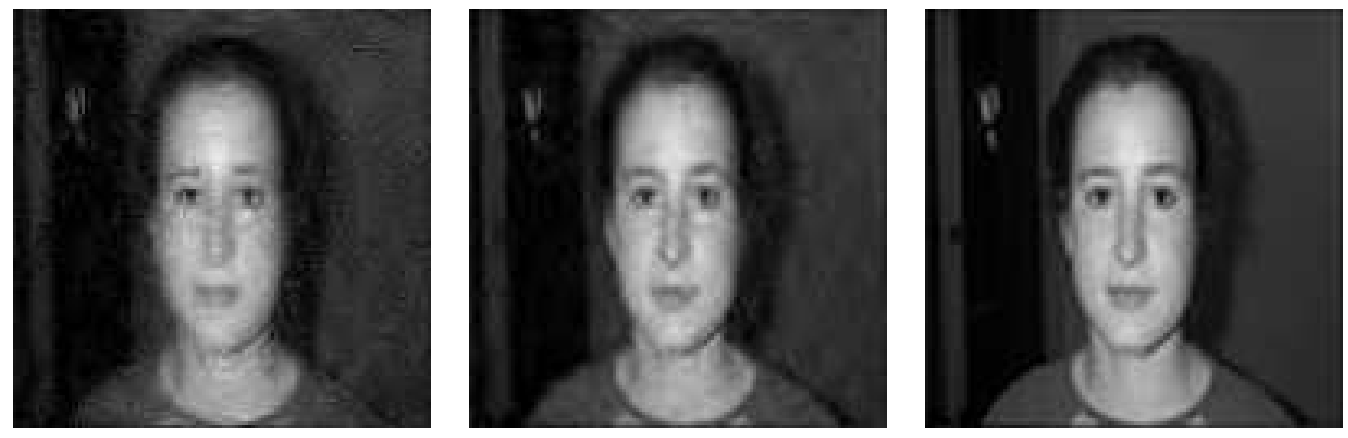

(a) Image reconstruction em- (b) Image reconstruction em- (c) Image reconstruction employing EEP- $\Phi$. ploying TSWCS. ploying UEP- $\Phi$.

Figure 4.3: Visual performance comparison of various CS image reconstructions on sample image PPL2. Here $m=4000$ and $L=100$.

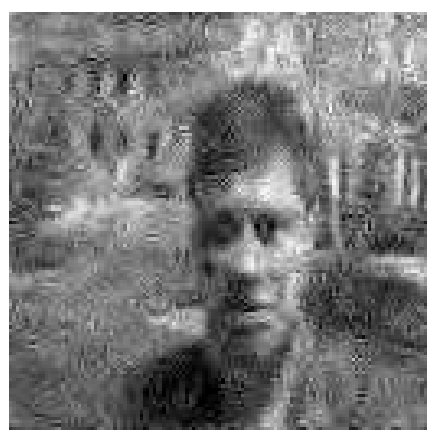

(a) Image reconstruction em-

Figure 4.4: Visual performance comparison of various CS image reconstructions on sample image PPL10. Here $m=4000$ and $L=100$.

+ UEP- $\Phi$, can further improve the reconstruction quality of TSWCS. Therefore, to extend our performance evaluation, we compare the performance of our algorithm with TSWCS, TSWCS + UEP- $\Phi$, and EEP employing a sparse and dense EEP- $\Phi_{\mathrm{S}}$ in Figure 4.9. Further, we compare the CPU runtime of our algorithm with that of TSWCS in Figure 4.10.

Figure 4.9 shows that our proposed algorithm significantly improves the image reconstruction quality compared to TSWCS, while it decreases the CPU+ runtime 

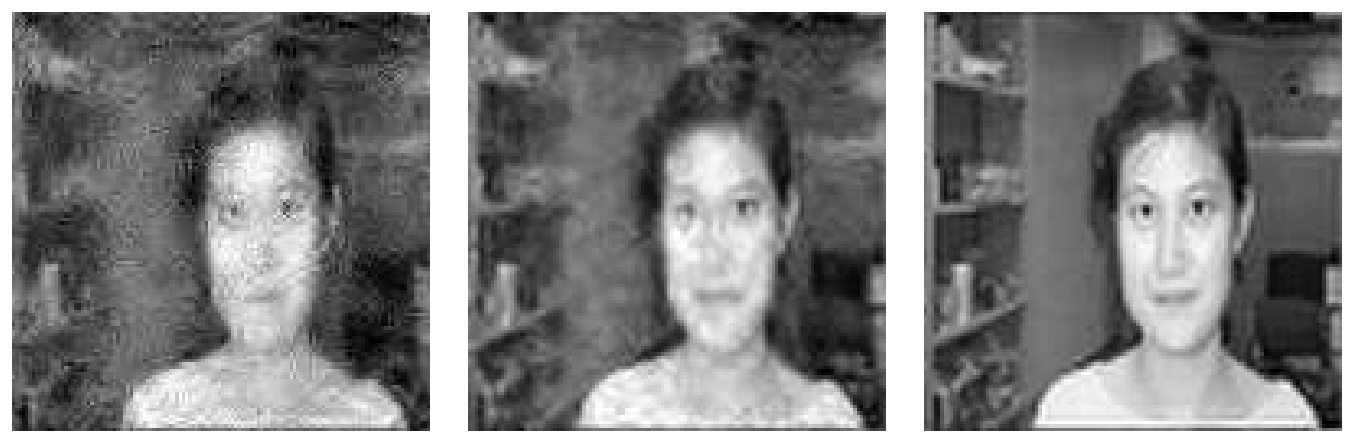

(a) Image reconstruction em- (b) Image reconstruction em- (c) Image reconstruction employing EEP- $\Phi$. ploying TSWCS. ploying UEP- $\Phi$.

Figure 4.5: Visual performance comparison of various CS image reconstructions on sample image PPL11. Here $m=4000$ and $L=100$.

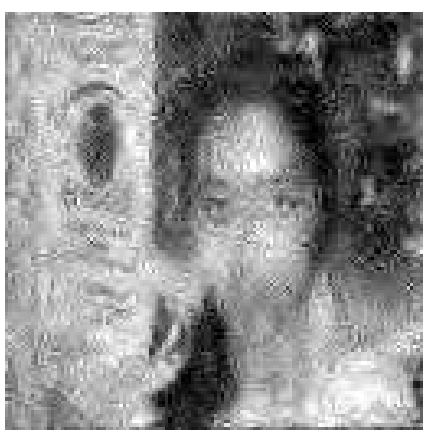

(a) Image reconstruction em-

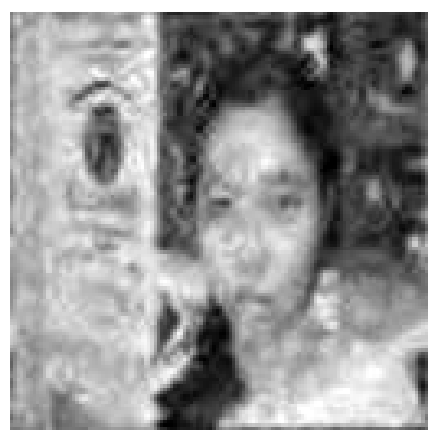

(b) Image reconstruction employing TSWCS.

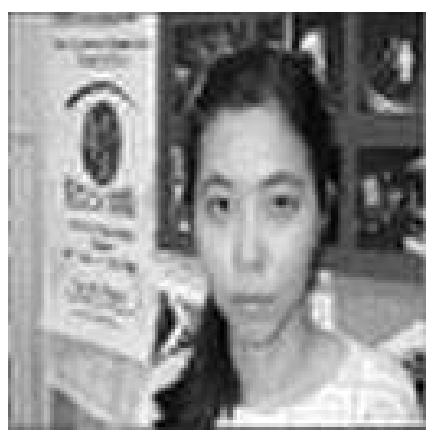

(c) Image reconstruction employing UEP- $\Phi$. ploying EEP- $\Phi$.

$$
\text { ploying TSWes. }
$$

Figure 4.6: Visual performance comparison of various CS image reconstructions on sample image PPL12. Here $m=4000$ and $L=100$.

as observed in Figure 4.10. We can also see that employing a sparse EEP- $\Phi$ results in a slight performance loss compared to a dense $\Phi$. However, employing a sparse $\Phi$ allows us to design UEP- $\Phi$ since a dense $\Phi$ uniformly captures all coefficients and no UEP may be provided. We emphasize that in contrast to TSWCS, the decoder remains intact when UEP- $\Phi$ is employed and the complexity of image reconstruction is equivalent to that of EEP- $\Phi$.

From Figure 4.9 we can see that combining TSWCS and UEP- $\Phi$ provides recon- 

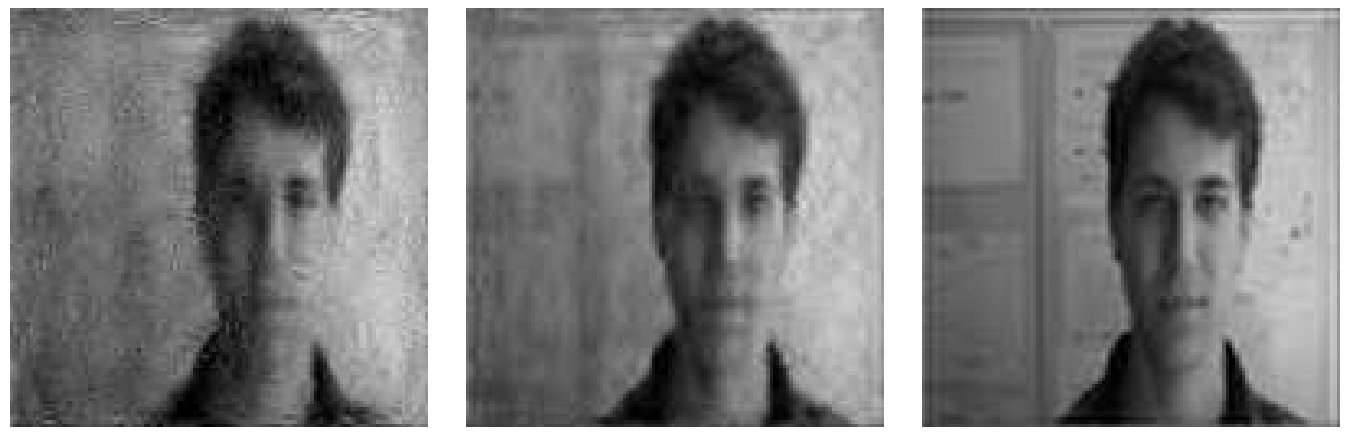

(a) Image reconstruction em- (b) Image reconstruction em- (c) Image reconstruction employing EEP- $\Phi$. ploying TSWCS. ploying UEP- $\Phi$.

Figure 4.7: Visual performance comparison of various CS image reconstructions on sample image PPL17. Here $m=4000$ and $L=100$.
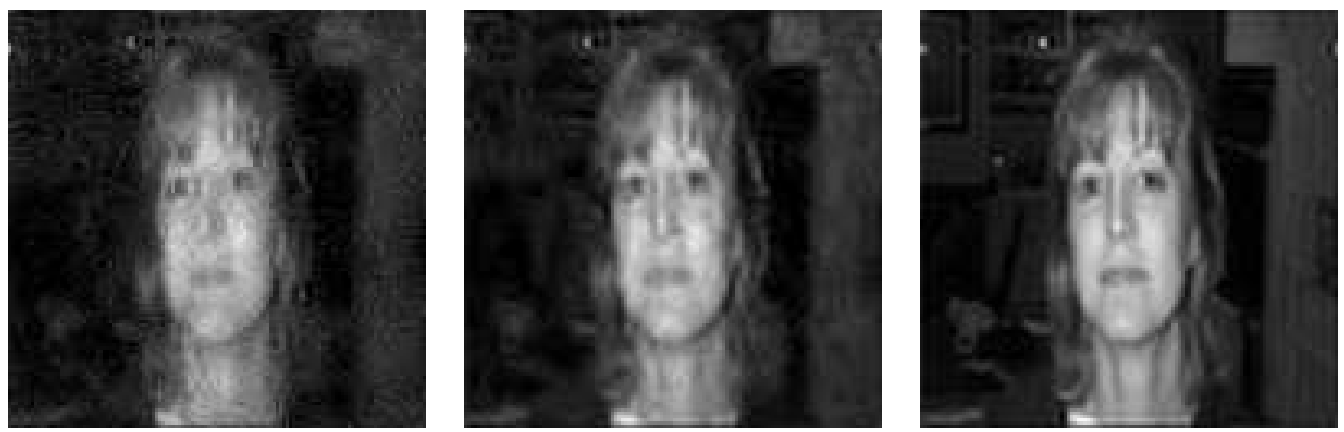

(a) Image reconstruction em- (b) Image reconstruction em- (c) Image reconstruction employing EEP- $\Phi$. ploying TSWCS. ploying UEP- $\Phi$.

Figure 4.8: Visual performance comparison of various CS image reconstructions on sample image PPL20. Here $m=4000$ and $L=100$.

struction quality better than that obtained by the TSWCS. We visually compare the quality of the reconstructed images using $T S W C S+U E P-\Phi$, TSWCS and UEP- $\phi$ for whole image when $M=4000$ in Figure 4.11. The reconstruction quality obtained by combining UEP- $\Phi$ with TSWCS is however better than our algorithm only for very small values of $\mathrm{M}$ as could be seen in Figures 4.9 and 4.11 . 


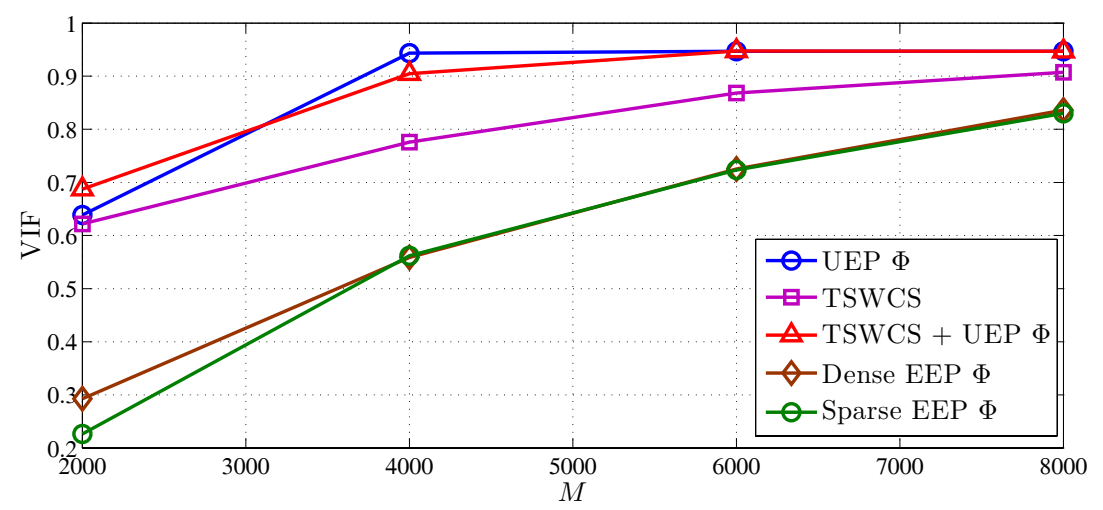

Figure 4.9: Image reconstruction performance comparison of our proposed algorithm versus existing algorithms.

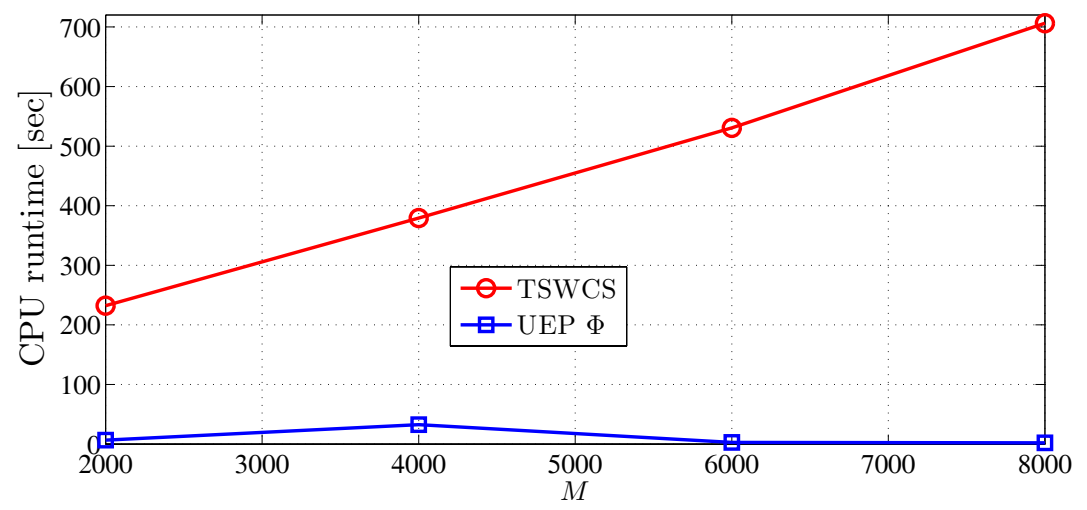

Figure 4.10: The CPU runtime comparison of proposed algorithm and TSWCS versus $M$.

\subsection{Prioritized ROI protection}

Providing more protection for the whole image coefficients has been discussed in Section 4.1. In this section we are interested in providing further protection for the ROI while the whole image coefficients are protected as discussed in Section 4.1. 

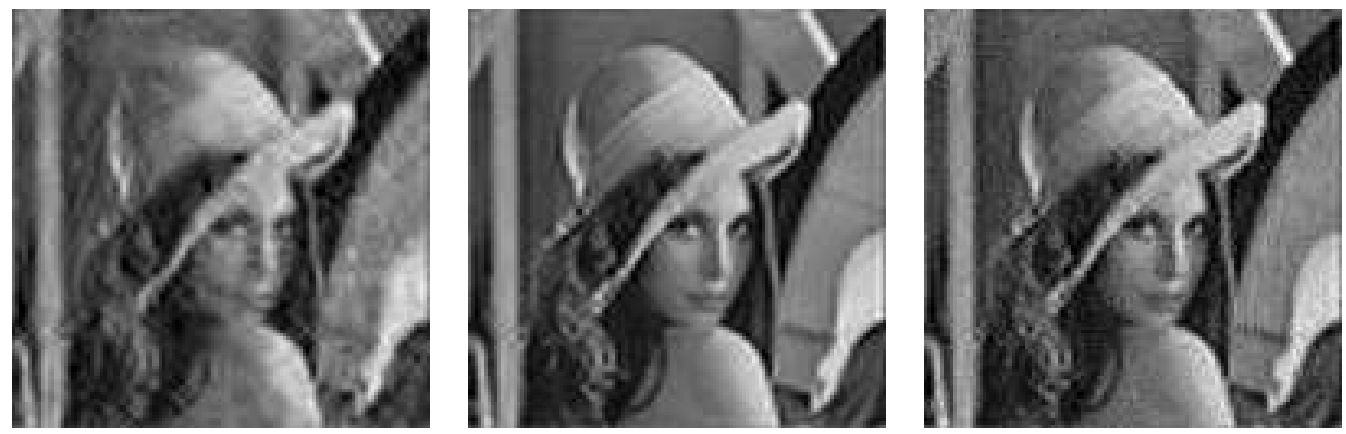

(a) Reconstruction employing (b) Reconstruction employing (c) Combined UEP- $\Phi$ encoding TSWCS.

UEP- $\Phi$. and TSWCS reconstruction.

Figure 4.11: Visual performance comparison of various CS schemes for images for $m=4000$.

\subsection{1 wavelet trees}

One of the characteristics of the wavelet transform that makes it very important is the temporal information it provides. In other words, the wavelet transform captures the frequency and location information of each image coefficient. The frequency information provided by the wavelet coefficients was mainly used for the application discussed in Section4.1. The location information provided by the wavelet transform is implemented in this section to provide more protection to a part of an image identified to be the region of interest (ROI). The importance of providing more importance to the ROI of a given imagery has been discussed in Sections 3.1 and 3.2.

The transform coefficients vector, $\underline{s}$, could be represented in the form of a tree structure known as the wavelet tree. Figure 4.12(a) shows a three level wavelet decomposition on Lena image and Figure 4.12(b) shows the wavelet tree structure. The block represented by $A$ on top left contains the approximate coefficients which are the largest transform coefficients. The blocks represented with $l=1, l=2$ and $l=3$ contain the transform coefficients in the first, second and third decomposition levels.

As it is shown in Figure 4.12(b), each coefficient in the second and higher decompo- 
sition levels has four children in the next lower decomposition level. In Figure4.12(b), for example, it could be seen that each coefficient in the third decomposition level has four children in the second decomposition level. Each of these four coefficients in the second level has four children in the first decomposition level. This process could also be described in terms of down sampling process starting from the first decomposition level as will be discussed in the next subsection.

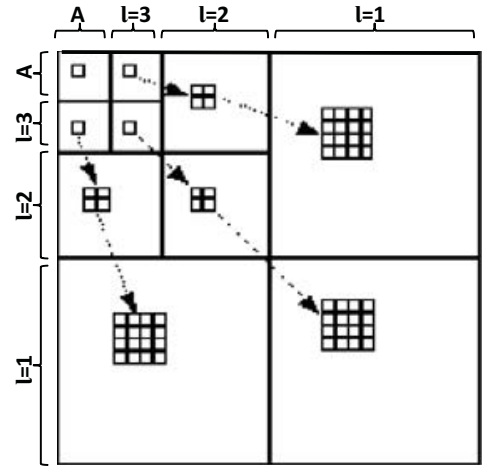

(a) Third level decomposition.

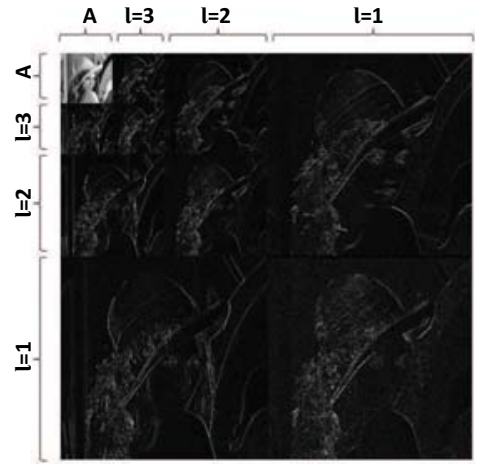

(b) wavelet tree.

Figure 4.12: Wavelet decomposition of Lena image and wavelet tree.

\subsubsection{Protecting ROI transform coefficients}

In order to provide better reconstruction quality for the ROI of a given imagery, the transform coefficients corresponding to this ROI must be incorporated into more measurements during the encoding process. As a result, the transform coefficients of a given imagery which correspond to the ROI must first be identified to provide better reconstruction quality for the ROI.

The wavelet decomposition process could be viewed as a filtering and a down sampling process as shown in Figure 4.2. Assume a particular area of an image (represented in the spatial domain) is identified to be the region of interest. Let the size of this ROI be $8 \times 8$. In the first decomposition level, the image is filtered and the columns and rows are down sampled by a factor of two. The ROI will, 
hence, be represented by transform coefficients of size $4 \times 4$ located in each of the three blocks containing transform coefficients of level one decomposition $(l=1$ in the Figure $4.12(\mathrm{~b}))$. This process is repeated resulting in a $2 \times 2$ transform coefficients representing the ROI in the second decomposition level $(l=2)$. In Figure 4.12(b), this process is repeated one more time resulting in a single transform coefficient in each of the three blocks containing transform coefficients of the third decomposition level $(l=3)$.

For our application, a Matlab program is developed which tracks the transform coefficients in the transform coefficient vector, $\underline{s}$, using the coefficients' relationship as discussed above. The significant transform coefficients located at the beginning of $\underline{s}$ are considered to be $M I C \mathrm{~s}$ as discussed in Section4.1. This provides a good reconstruction quality for all the image parts as a whole. Further more, the transform coefficients that correspond to the ROI are also treated as $M I C$ s to provide further protection for the ROI. The measurement matrix $\phi_{i, j}$ is thus constructed so as an entry at in the $j^{\text {th }}$ column of $\Phi$ for any row is made to be a non-zero with a probability of $P_{1}$ or $P_{2}$ depending on whether $s_{j}$ is an MIC or an LIC as discussed in Section 3.2.

Table 4.2 shows the simulation results obtained implementing this algorithm on our test images. The ROI is assumed to be the part of the sample images identified to be the main subject as shown in Figure 3.1. The parameters used for these simulations are: $L=100$ and $k_{M}=5$ while the $M I C$ s are set to be the first 1024 coefficients and those transform coefficients that represent the ROI.

In Table 4.2, the VIF values presented under the title ' $M I C$ ' are measured for the $R O I$ s of the images; Whereas, those under ' $L I C$ ' are measured for the rest part of the image. The entries under the titles 'UEP' and 'TSWCS' are obtained using the algorithms proposed in Section 4.1 and contribution [5] respectively. These values are those presented in Table 4.1. They are presented here again for convenience.

As it could be seen from Table 4.2, the ROI has been reconstructed with a better 
quality for all the images while the quality of the rest of the image is not significantly degraded. Sample images are shown in Figures 4.13, 4.14 and 4.15 for subjective comparison.

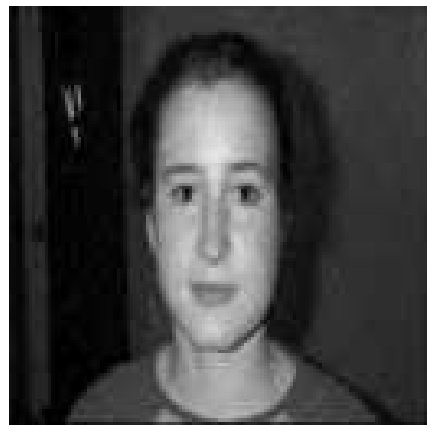

(a) TSWCS reconstruction.

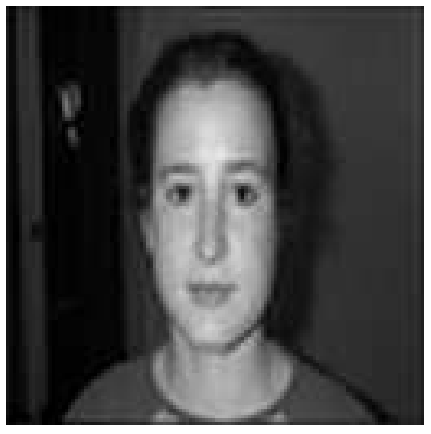

(b) UEP- $\Phi$ for whole image.

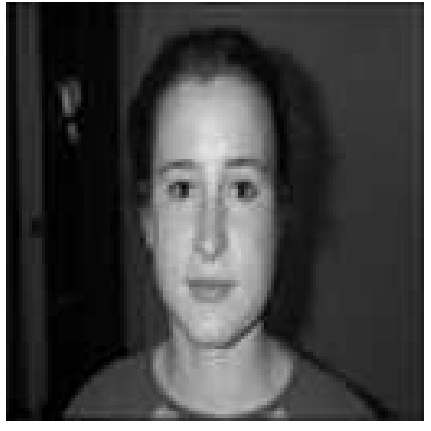

(c) UEP- $\Phi$ with prioritized ROI reconstruction.

Figure 4.13: Visual performance comparison of various CS image reconstructions for sample image PPL2. Here $m=8000$ and $L=100$.

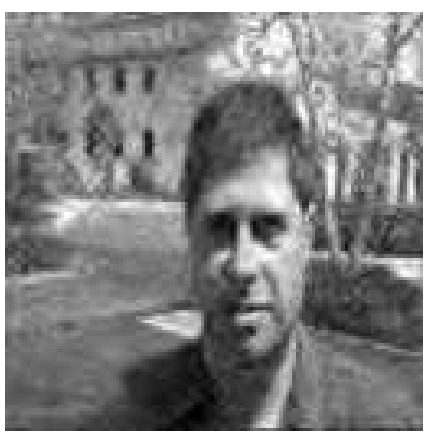

(a) TSWCS reconstruction.

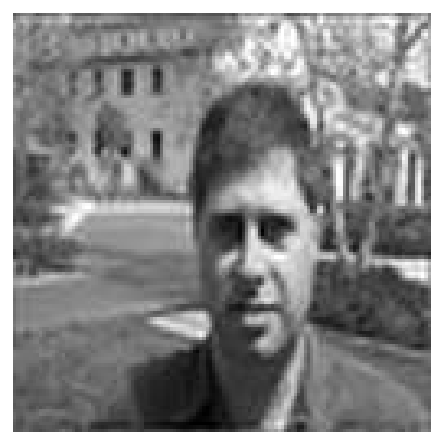

(b) UEP- $\Phi$ for whole image.

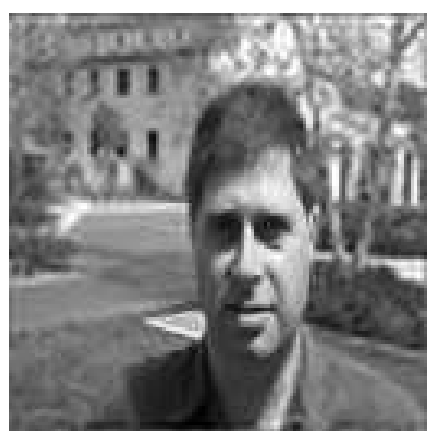

(c) UEP- $\Phi$ with prioritized ROI reconstruction.

Figure 4.14: Visual performance comparison of various CS image reconstructions for sample image PPL10. Here $m=8000$ and $L=100$.

From Figures 4.13 to 4.18 , we can see that the region of interest (ROI) has been recovered with an even better quality with the prioritized ROI protection method, while the the background is recovered with a quality as good as UEP- $\phi$ for whole 


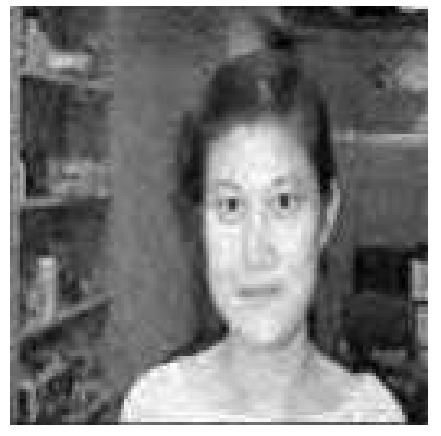

(a) TSWCS reconstruction.

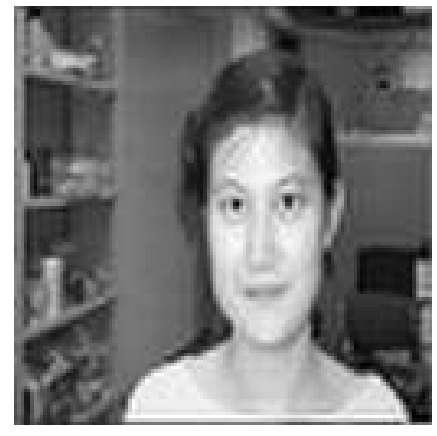

(b) UEP- $\Phi$ for whole image.

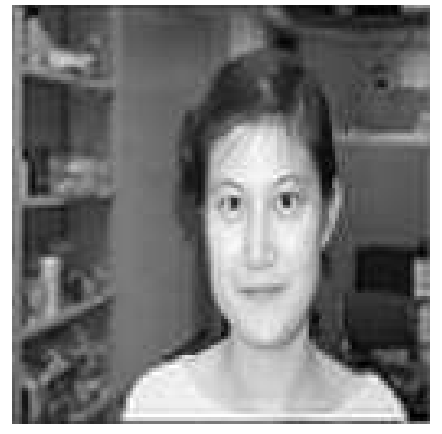

(c) UEP- $\Phi$ with prioritized ROI reconstruction.

Figure 4.15: Visual performance comparison of various CS image reconstructions for sample image PPL11. Here $m=8000$ and $L=100$.

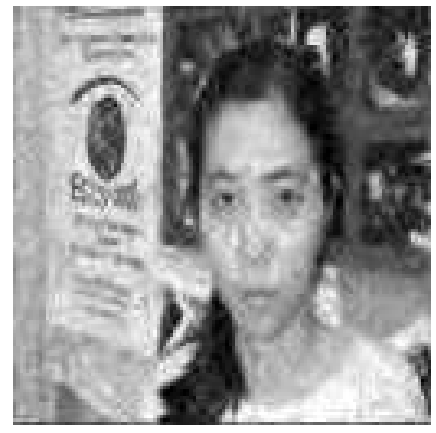

(a) TSWCS reconstruction.

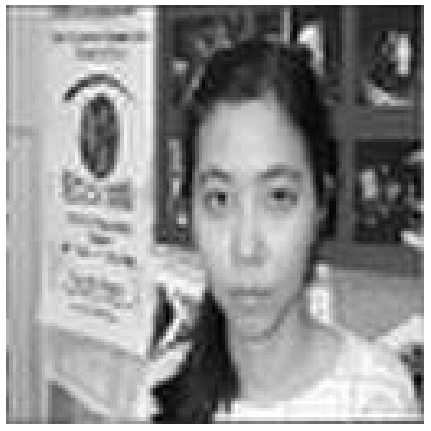

(b) UEP- $\Phi$ for whole image.

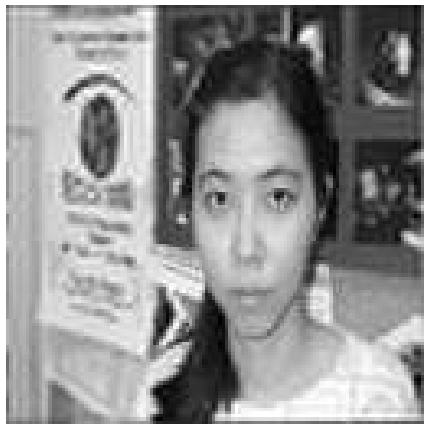

(c) UEP- $\Phi$ with prioritized ROI reconstruction.

Figure 4.16: Visual performance comparison of various CS image reconstructions for sample image PPL12. Here $m=8000$ and $L=100$.

image (Section 4.1). It has further been shown in Section 4.1 that UEP- $\phi$ for whole image results in a reconstruction quality better than most state of the art reconstruction methods. As a result, the prioritized ROI protection method recovers the background with a quality better than most state of the art CS reconstruction schemes and recovers the ROI with an even better quality. 


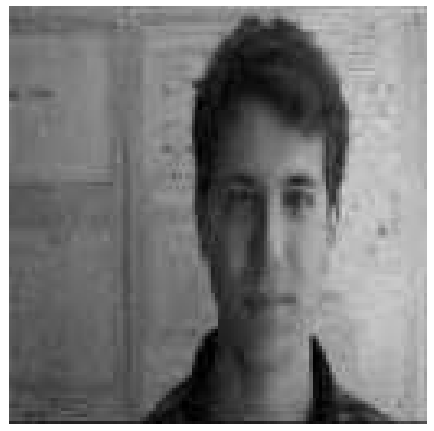

(a) TSWCS reconstruction.

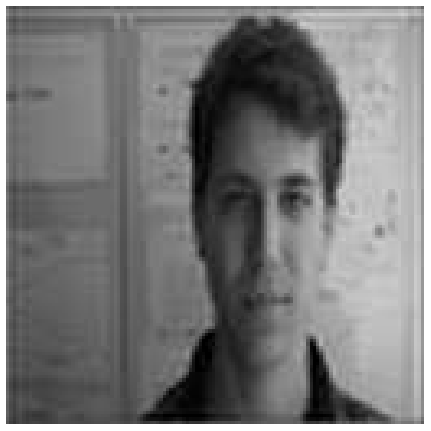

(b) UEP- $\Phi$ for whole image.

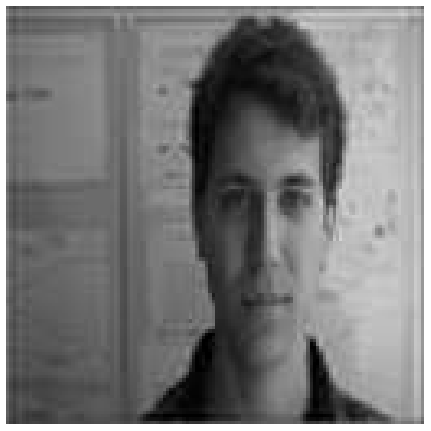

(c) UEP- $\Phi$ with prioritized ROI reconstruction.

Figure 4.17: Visual performance comparison of various CS image reconstructions for sample image PPL17. Here $m=8000$ and $L=100$.

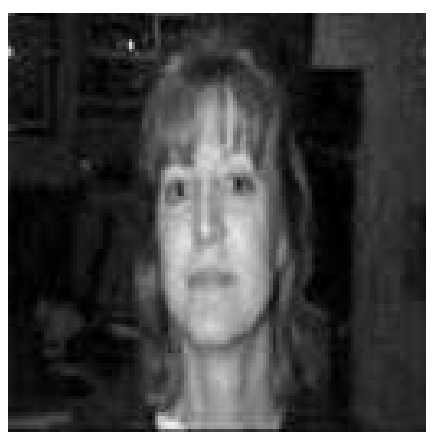

(a) TSWCS reconstruction.

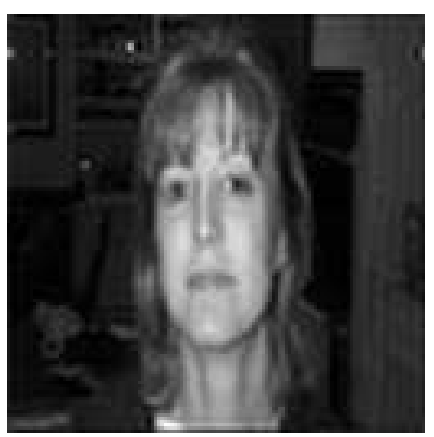

(b) UEP- $\Phi$ for whole image.

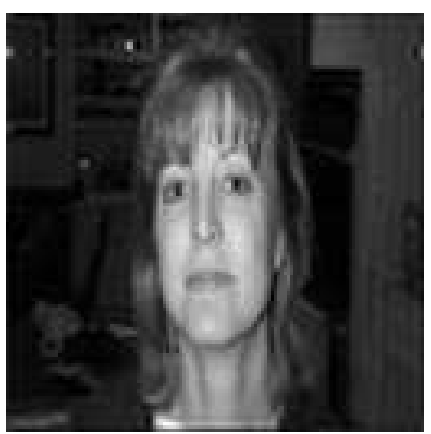

(c) UEP- $\Phi$ with prioritized ROI reconstruction.

Figure 4.18: Visual performance comparison of various CS image reconstructions for sample image PPL20. Here $m=8000$ and $L=100$. 
Table 4.2: VIF of images reconstructed by UEP- $\phi$ for whole image, TSWCS and prioritized ROI protection algorithms.

\begin{tabular}{|r|r|r|r|r|r|r|}
\hline Images & P2 & P10 & P11 & P12 & P17 & P20 \\
\hline \multicolumn{7}{|c|}{$\mathrm{M}=6000$} \\
\hline MIC & 0.994 & 0.996 & 0.984 & 0.976 & 0.997 & 0.986 \\
\hline LIC & 0.983 & 0.944 & 0.954 & 0.928 & 0.98 & 0.949 \\
\hline Total & 0.985 & 0.947 & 0.964 & 0.925 & 0.975 & 0.969 \\
\hline TSWCS & 0.956 & 0.771 & 0.876 & 0.818 & 0.914 & 0.902 \\
\hline UEP & 0.98 & 0.932 & 0.963 & 0.942 & 0.969 & 0.963 \\
\hline \multicolumn{7}{|c|}{} \\
\hline MIC & 0.998 & 0.996 & 0.993 & 0.996 & 1.000 & 0.999 \\
\hline LIC & 0.987 & 0.944 & 0.963 & 0.947 & 0.982 & 0.963 \\
\hline Total & 0.989 & 0.947 & 0.974 & 0.952 & 0.979 & 0.981 \\
\hline TSWCS & 0.985 & 0.836 & 0.922 & 0.876 & 0.957 & 0.934 \\
\hline UEP & 0.98 & 0.932 & 0.963 & 0.942 & 0.969 & 0.963 \\
\hline
\end{tabular}




\section{CHAPTER 5}

\section{CONCLUSIONS}

In this thesis work, we proposed to incorporate the unequal error protection (UEP) ideas, from error correction codes, into compressive sensing (CS) algorithm for image reconstruction. We proposed a novel measurement matrix construction method, for CS encoding phase, by which a higher protection can be assigned to more important coefficients.

It is well-known that images usually have at least one main subject which draws the viewers attention and conveys the image's information. Hence, in our first study, the main subject area is assumed to be more important than the rest of the image. Therefore, we proposed to capture this area in more number of measurements by implementing unequal compressive sampling to increase its reconstruction accuracy. We observed that this significantly improves the reconstruction quality of the main subject, while resulting in slight degradation in the less important areas.

In our second approach, we make use of the well-known fact that the sparse representation of an image has most of its significant coefficients (that convey the most of the image's information) concentrated at the beginning of the transform coefficients' vector. Therefore, we proposed to generate samples from the signal such that these more important coefficients of the sparse signal are more strongly captured by the measurements. We showed that this would significantly improve the overall image reconstruction quality. Furthermore, we showed that our proposed algorithm surpass exiting CS algorithms for image reconstruction while providing low complexity.

Finally, we employ the known tree structure of wavelet transform coefficients to lo- 
cate the coefficients that correspond to the main subject in the transform coefficients' vector. We then propose to capture the more informative transform coefficients (which are located at the beginning of the transform coefficients' vector) and also the transform coefficients that correspond to the main subject more strongly than the rest of the transform coefficients. We, then, showed that this would provide an improved reconstruction quality for the whole image while the main subject is reconstructed with an even better quality.

This work could be broadened by considering multiple main subjects and multiple levels of importance. Employing saliency map to find the main subject could also be experimented with as it could trick the visual system into perceiving the reconstructed image to be of more quality. This is because the transition between the more important and less important image coefficient would be less visible after CS reconstruction. Another interesting extension would be the integration of our first algorithm (Chapter with the single pixel camera [17]. 


\section{BIBLIOGRAPHY}

[1] D. Donoho, "Compressed sensing," IEEE Transactions on Information Theory, vol. 52, p. 12891306, Apr. 2006.

[2] B. OHanen and M. Wisan, "Jpeg compression," Dec. 2005.

[3] G. K. Wallace, "The jpeg still picture compression standard," IEEE Transactions on Consumer Electronics, vol. 38, pp. 18-34, Feb. 1992.

[4] M. Lustig, D. Donoho, J. Santos, and J. Pauly, "Compressed sensing mri," IEEE Signal Processing Magazine, vol. 25, no. 2, pp. 72 -82, 2008.

[5] L. He and L. Carin, "Exploiting structure in wavelet-based bayesian compressive sensing," IEEE Transactions on Signal Processing, vol. 57, pp. 3488-3497, September 2009.

[6] S. S. D. Baron and R. G. Baraniuk, "Bayesian compressive sensing via belief propagation," IEEE Transactions on Signal Processing, vol. 58, pp. 269-280, 2010 Jan.

[7] N. Rahnavard, B. Vellambi, and F. Fekri, "Rateless codes with unequal error protection property," IEEE Transactions on Information Theory, vol. 53, pp. 15211532, April 2007.

[8] A. B. Victor Sanchez and M. K. Mandal, "Prioritized region of interest coding in jpeg2000," IEEE Transactions on Circuits and Systems for Video Technology, vol. 14, pp. 1149 - 1155, Sept. 2004. 
[9] E. Candes and M. Wakin, "An introduction to compressive sampling [a sensing/sampling paradigm that goes against the common knowledge in data acquisition]," IEEE Signal Processing Magazine, vol. 25, pp. 21 - 30, Mar. 2008.

[10] E. Candes, J. Romberg, and T. Tao, "Robust uncertainty principles: exact signal reconstruction from highly incomplete frequency information," IEEE Trans. on Information Theory, vol. 52, pp. 489-509, Feb. 2006.

[11] E. Candes and T. Tao, "Near-optimal signal recovery from random projections: Universal encoding strategies?," IEEE Transactions on Information Theory, vol. 52, no. 12 , pp. $5406-5425,2006$.

[12] S. S. Chen, D. L. Donoho, Michael, and M. A. Saunders, "Atomic decomposition by basis pursuit," SIAM Journal on Scientific Computing, vol. 20, pp. 33-61, 1998.

[13] C. Vu and D. Chandler, "Main subject detection via adaptive feature selection," IEEE International Conference on Image Processing, pp. 3101 - 3104, November 2009.

[14] R. Berinde and P. Indyk, "Sparse recovery using sparse random matrices," MIT, Cambridge, MA, Tech. Rep. MIT-CSAIL-TR-2008-001, 2008.

[15] N. Rahnavard and F. Fekri, "Generalization of rateless codes for unequal error protection and recovery time: Asymptotic analysis," IEEE International Symposium on Information Theory, pp. 523-527, July 2006.

[16] H. Sheikh and A. Bovik, "Image information and visual quality," IEEE Transactions on Image Processing, vol. 15, no. 2, pp. 430 -444, 2006.

[17] M. D. M. Duarte and R. Baraniuk, "Single pixel imaging via compressive sampling," IEEE Signal Processing Magazine, vol. 25, pp. 83 - 91, Mar. 2008. 
[18] R. Baraniuk, V. Cevher, M. Duarte, and C. Hegde, "Model-based compressive sensing," IEEE Transactions on Information Theory, vol. 56, no. 4, pp. 1982 $-2001,2010$.

[19] W. Wang, M. Garofalakis, and K. Ramchandran, "Distributed sparse random projections for refinable approximation," International conference on Information processing in sensor networks, pp. 331-339, 2007.

[20] D. Baron, S. Sarvotham, and R. Baraniuk, "Bayesian compressive sensing via belief propagation," IEEE Transactions on Signal Processing, vol. 58, no. 1, pp. $269-280,2010$.

[21] J. Tropp and A. Gilbert, "Signal recovery from random measurements via orthogonal matching pursuit," IEEE Transactions on Information Theory, vol. 53, pp. 4655-4666, Dec. 2007.

[22] E. van den Berg and M. P. Friedlander, "Probing the pareto frontier for basis pursuit solutions," SIAM Journal on Scientific Computing, vol. 31, no. 2, pp. 890-912, 2008.

[23] F. Zhang and H. D. Pfister, "On the iterative decoding of high-rate ldpc codes with applications in compressed sensing." 2009.

[24] A. P. Bradley and F. W. M. Stentiford, "Jpeg 2000 and region of interest coding," Digital Image Computing Techniques and Applications (DICTA), Jan. 2002.

[25] J. A. C. Christopoulos and M. Larsson, "Efficient methods for encoding regions of interest in the upcoming jpeg2000 still image coding standard," IEEE Signal Processing Letters, vol. 7, pp. 247-249, Jan. 2000. 


\section{APPENDIX A}

\section{Supplementary for Chapter 3}

In Chapter 3, the DCT was used as a basis of sparsity. Results obtained when the two-dimensional DWT is used as a basis of sparsity are presented below in Table A.1.

Table A.1: VIF of CS reconstruction performance employing EEP- and UEP- $\Phi$ s for wavelet $\psi$.

\begin{tabular}{|c|c|c|c|c|c|c|c|c|}
\hline \multicolumn{3}{|c|}{ Images } & $\mathrm{P} 2$ & P10 & P11 & P12 & P17 & P20 \\
\hline \multicolumn{3}{|c|}{$\alpha$} & 0.18 & 0.13 & 0.17 & 0.21 & 0.17 & 0.19 \\
\hline \multirow[t]{4}{*}{ VIF } & EEP & Sparse & 0.81 & 0.44 & 0.61 & 0.59 & 0.77 & 0.72 \\
\hline & \multirow[t]{3}{*}{ UEP } & MIC & 1 & 0.94 & 0.98 & 0.97 & 1 & 1 \\
\hline & & LIC & 0.62 & 0.45 & 0.57 & 0.54 & 0.77 & 0.6 \\
\hline & & Total & 0.75 & 0.51 & 0.6 & 0.61 & 0.74 & 0.73 \\
\hline
\end{tabular}

The parameter values used in order to obtain the results presented in Table A.1 are similar to that used of Chapter 3 (i.e. $L=16, k_{M}=5, N=16,384$ and $M=6000$ ). The reconstructed images employing the EEP- and UEP- $\Phi$ s are shown in Figures A.1, A.2 and A.3 for images PPL2, PPL10 and PPL11. 


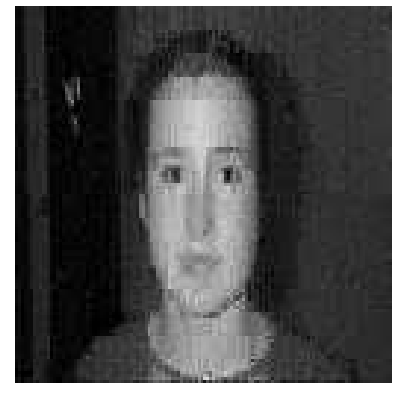

(a) Sparse EEP- $\Phi$

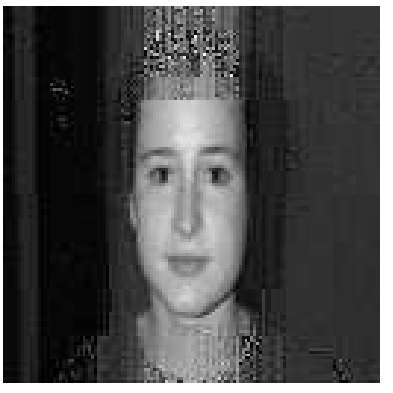

(b) UEP- $\Phi$

Figure A.1: Reconstruction quality comparison of UEP- $\phi$ versus dense and sparse EEP- $\phi$ s for sample image PPL2.

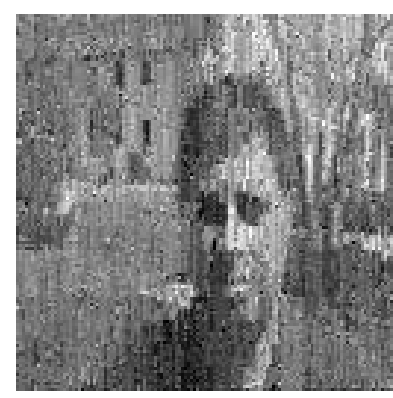

(a) Sparse EEP- $\Phi$

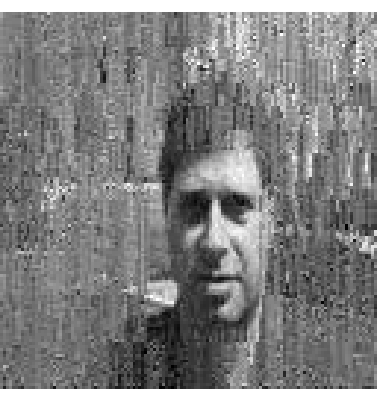

(b) UEP- $\Phi$

Figure A.2: Reconstruction quality comparison of UEP- $\phi$ versus dense and sparse EEP- $\phi$ s for sample image PPL10.

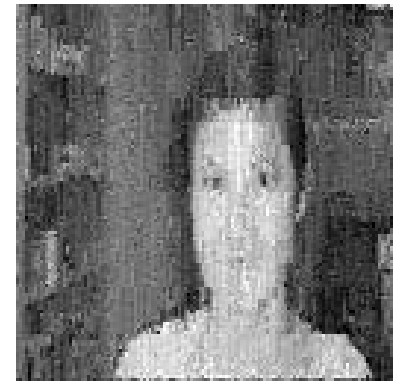

(a) Sparse EEP- $\Phi$

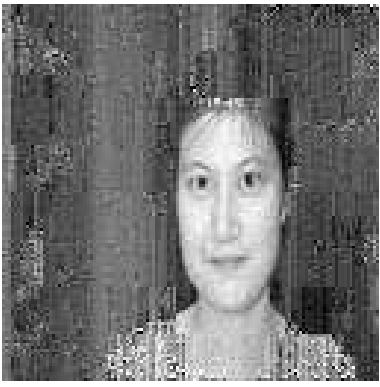

(b) UEP- $\Phi$

Figure A.3: Reconstruction quality comparison of UEP- $\phi$ versus dense and sparse EEP- $\phi$ s for sample image PPL11. 


\section{APPENDIX B}

\section{Supplementary for Chapter 4}

In Chapter 4, the two-dimensional DWT was used as a basis of sparsity. Results obtained when the DCT is used as a basis of sparsity are presented below in Table B.1.

Table B.1: VIF measure of the reconstructed images employing EEP- and UEP- $\Phi$ s for $\operatorname{DCT} \psi$.

\begin{tabular}{|c|c|c|c|c|c|c|c|}
\hline \multirow[t]{13}{*}{ VIF } & Images & $\mathrm{P} 2$ & P10 & $\mathrm{P} 11$ & $\mathrm{P} 12$ & $\mathrm{P} 17$ & P20 \\
\hline & \multicolumn{7}{|c|}{$M=2000$} \\
\hline & EP & 0.51 & 0.17 & 0.33 & 0.31 & 0.42 & 0.42 \\
\hline & UEP & 0.77 & 0.51 & 0.66 & 0.58 & 0.71 & 0.67 \\
\hline & \multicolumn{7}{|c|}{$\mathrm{M}=4000$} \\
\hline & $\mathrm{EP}$ & 0.74 & 0.4 & 0.57 & 0.51 & 0.65 & 0.6 \\
\hline & UEP & 0.91 & 0.79 & 0.87 & 0.81 & 0.86 & 0.83 \\
\hline & \multicolumn{7}{|c|}{$M=6000$} \\
\hline & EP & 0.83 & 0.6 & 0.74 & 0.66 & 0.78 & 0.75 \\
\hline & UEP & 0.92 & 0.8 & 0.88 & 0.82 & 0.86 & 0.83 \\
\hline & \multicolumn{7}{|c|}{$\mathrm{M}=8000$} \\
\hline & EP & 0.9 & 0.75 & 0.83 & 0.77 & 0.86 & 0.83 \\
\hline & UEP & 0.92 & 0.8 & 0.88 & 0.82 & 0.86 & 0.83 \\
\hline
\end{tabular}

The parameter values used in order to obtain the results presented in Table B.1 
are similar to that used of Chapter 4 (i.e. $\alpha \approx 0.25, L=100$, and $k_{M}=5$ and $N=16,384)$. The reconstructed images employing the EEP- and UEP- $\Phi$ s are shown in Figures B.1, B.2 and B.3 for images PPL2, PPL10 and PPL11 when $M=4000$.
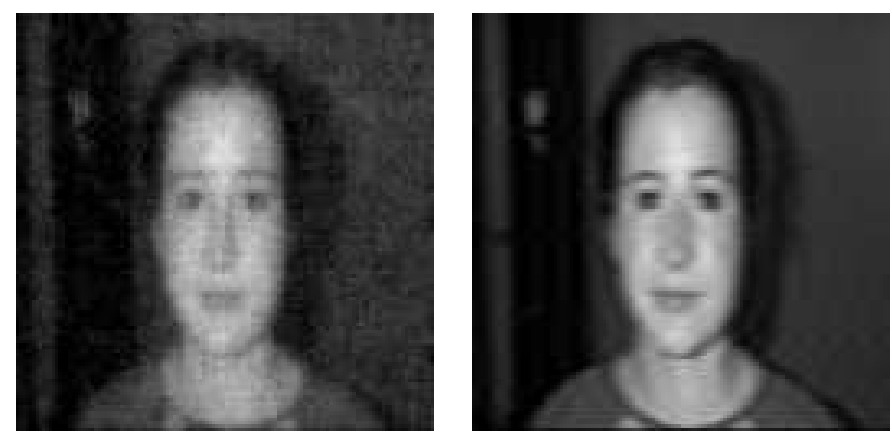

(a) Image reconstruction em- (b) Image reconstruction employing EEP- $\Phi$. ploying UEP- $\Phi$.

Figure B.1: Visual performance comparison of various CS image reconstructions on sample image PPL2.
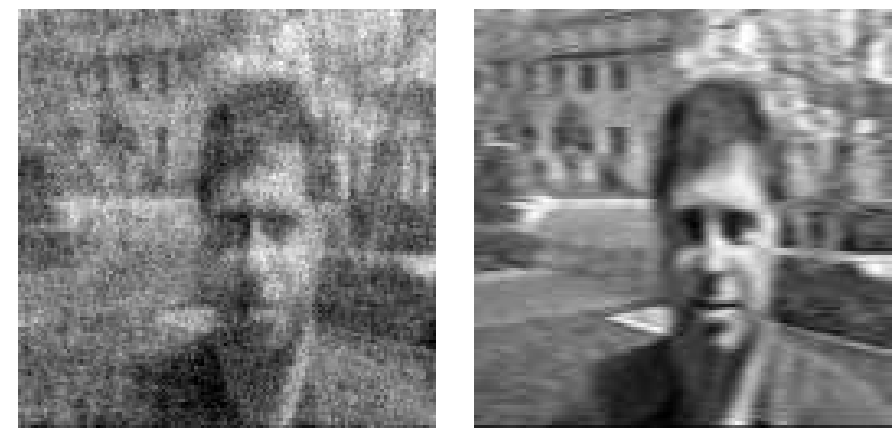

(a) Image reconstruction em- (b) Image reconstruction employing EEP- $\Phi$. ploying UEP- $\Phi$.

Figure B.2: Visual performance comparison of various CS image reconstructions on sample image PPL10.

\section{Supplementary images for Section 4.1, Table 4.1.}

The performance of EEP and TSWCS with UEP- $\phi$ for whole image using the sample images are shown below in Figures B.4 and B.5 when $M=6000$. 

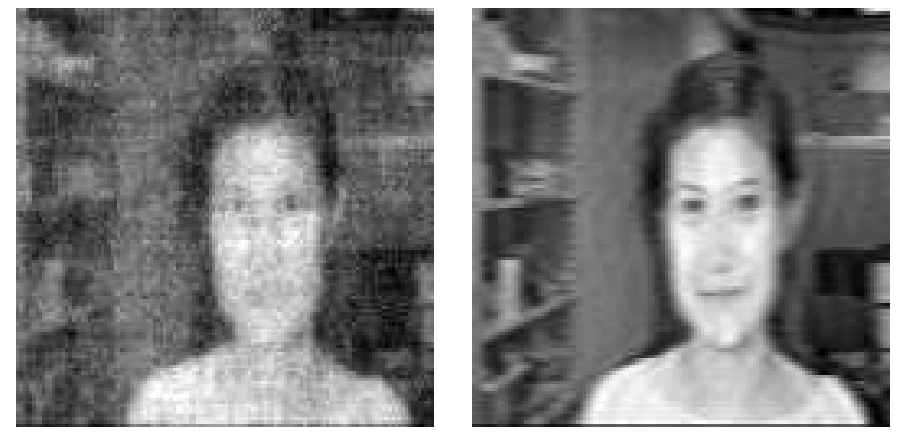

(a) Image reconstruction em- (b) Image reconstruction employing EEP- $\Phi$. ploying UEP- $\Phi$.

Figure B.3: Visual performance comparison of various CS image reconstructions on sample image PPL11.

\section{Supplementary images for Section 4.2, Table 4.2.}

The images reconstructed for $M=8000$ has been shown in Section 4.2, Figures B.6 and B.7. Images reconstructed for $M=6000$ are shown below. 

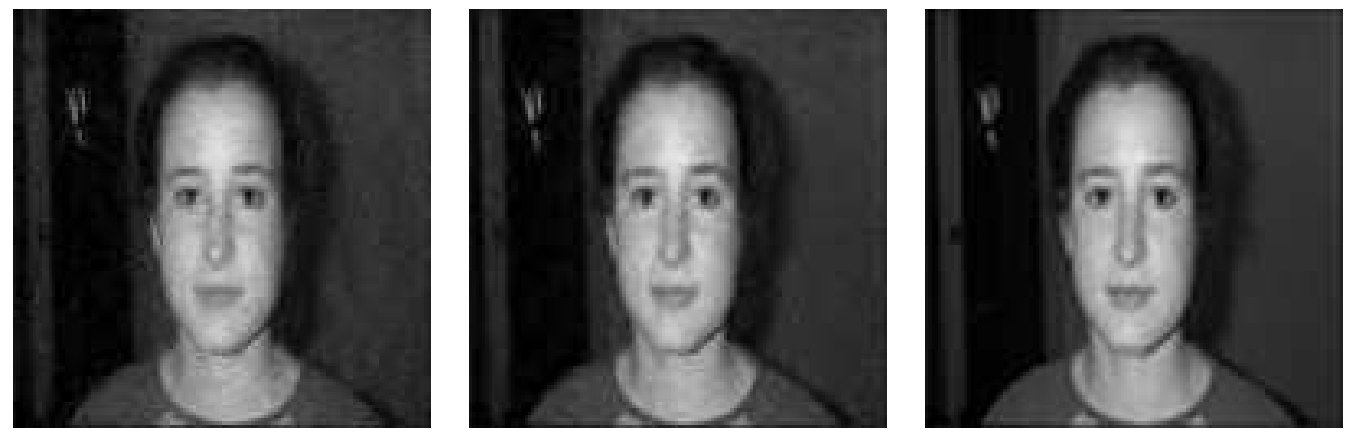

(a) Image reconstruction em- (b) Image reconstruction em- (c) Image reconstruction employing EEP- $\Phi$. ploying TSWCS. ploying UEP- $\Phi$.
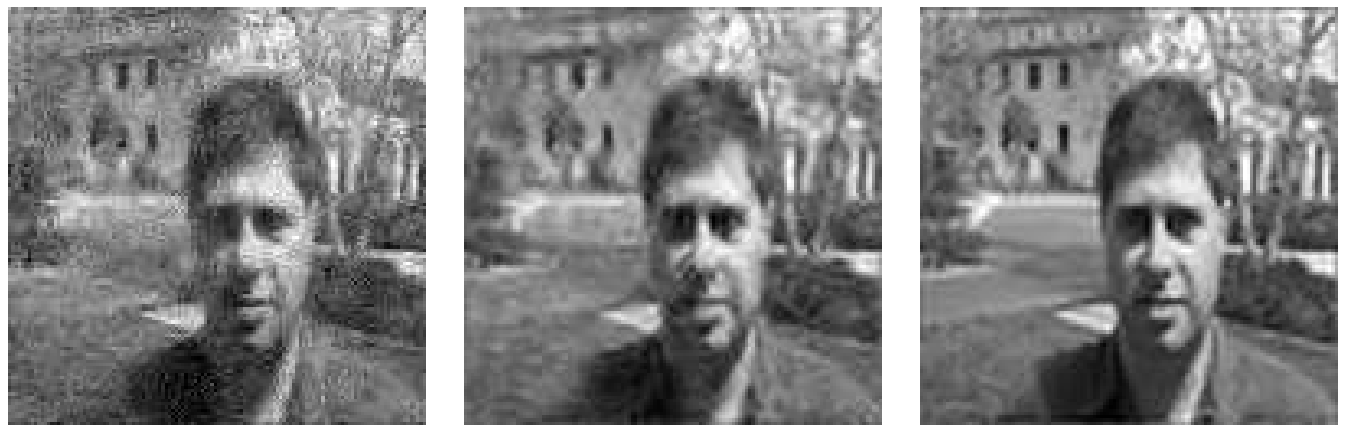

(d) Image reconstruction em-

(e) Image reconstruction em-

(f) Image reconstruction employing EEP- $\Phi$. ploying TSWCS. ploying UEP- $\Phi$.
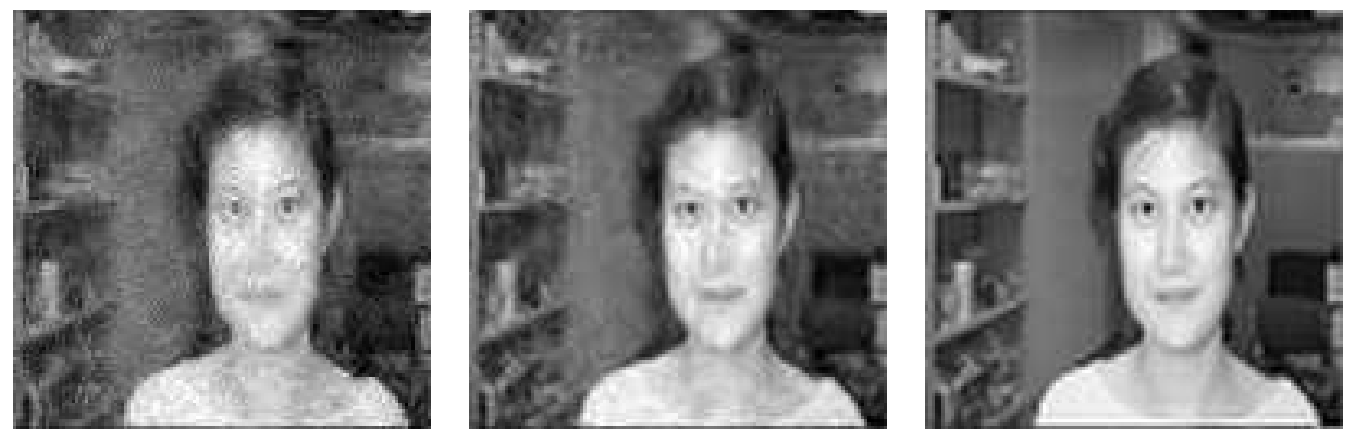

(g) Image reconstruction em- (h) Image reconstruction em- (i) Image reconstruction employing EEP- $\Phi$. ploying TSWCS. ploying UEP- $\Phi$.

Figure B.4: Visual performance comparison of various CS image reconstructions on sample images for $M=6000$. 

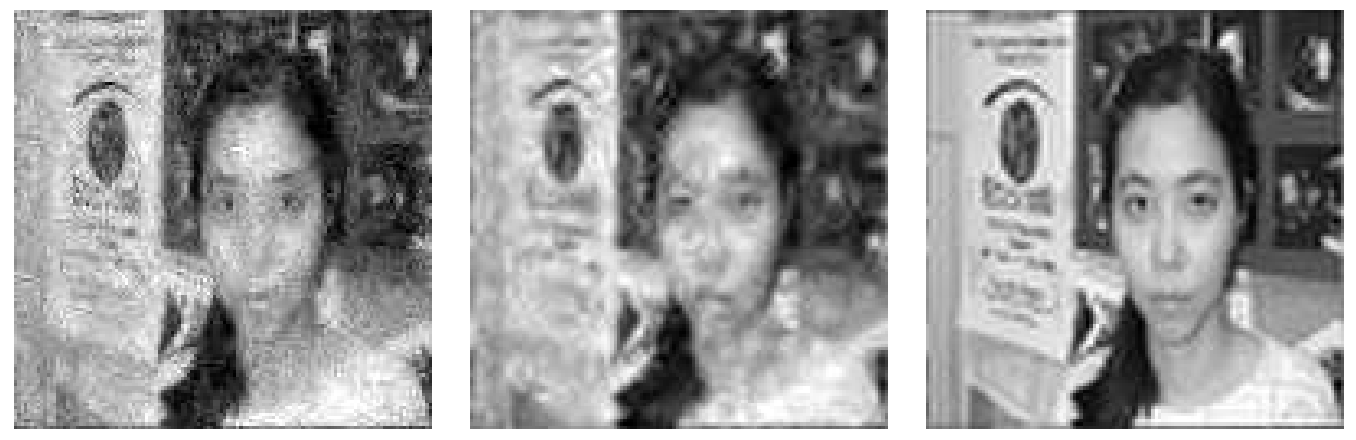

(a) Image reconstruction em- (b) Image reconstruction em- (c) Image reconstruction employing EEP- $\Phi$. ploying TSWCS.
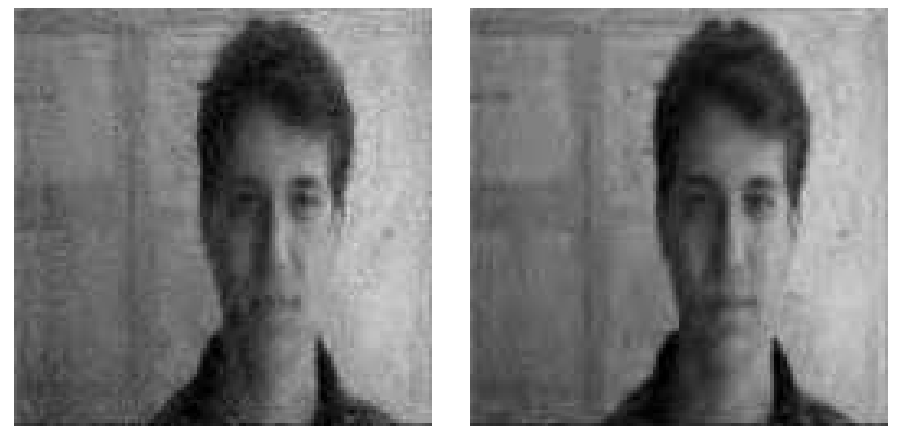
ploying UEP- $\Phi$.

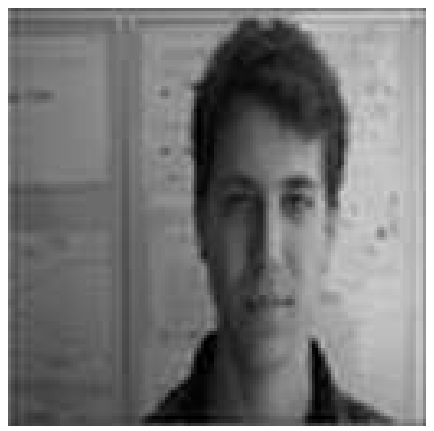

(d) Image reconstruction employing EEP- $\Phi$.

(e) Image reconstruction em-

(f) Image reconstruction em-

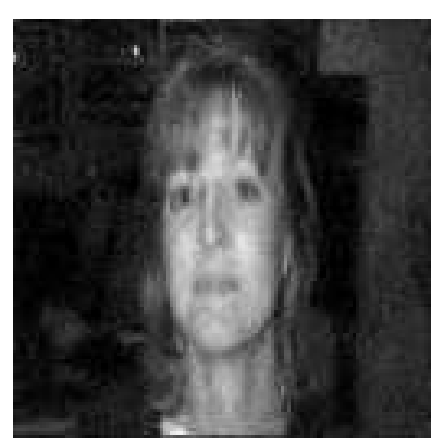
ploying TSWCS. ploying UEP- $\Phi$.
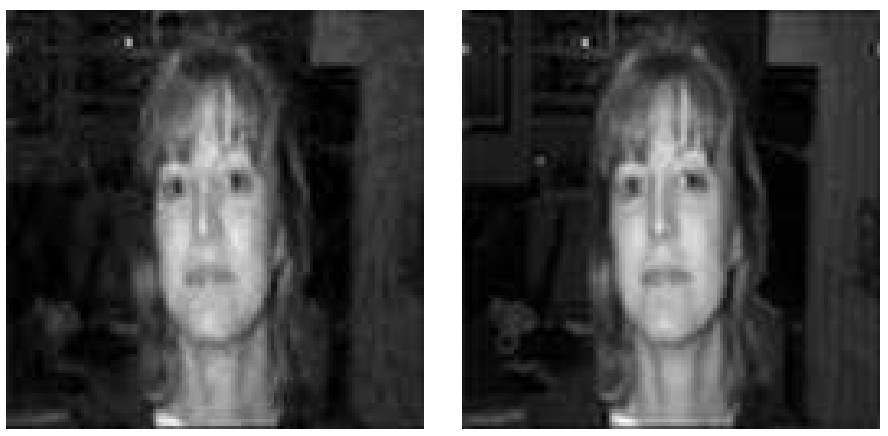

(g) Image reconstruction em- (h) Image reconstruction em- (i) Image reconstruction employing EEP- $\Phi$. ploying TSWCS. ploying UEP- $\Phi$.

Figure B.5: Visual performance comparison of various CS image reconstructions on sample images for $M=6000$. 


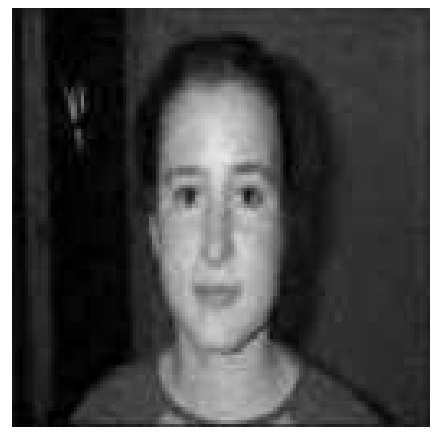

(a) TSWCS reconstruction.

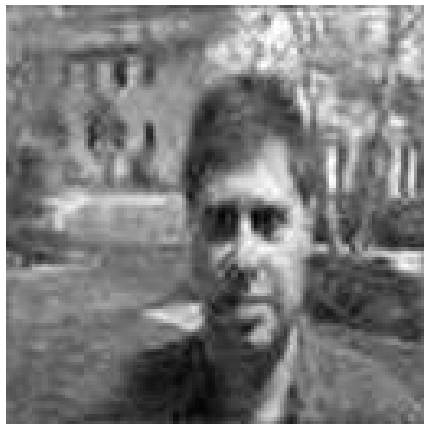

(d) TSWCS reconstruction.

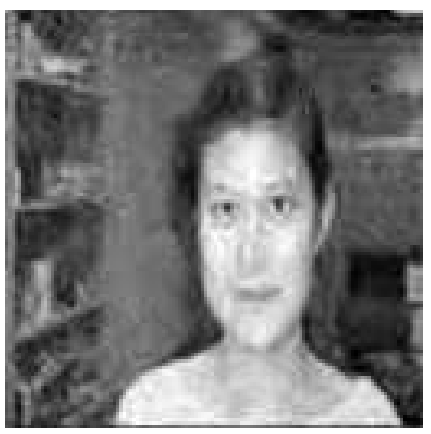

(g) TSWCS reconstruction.

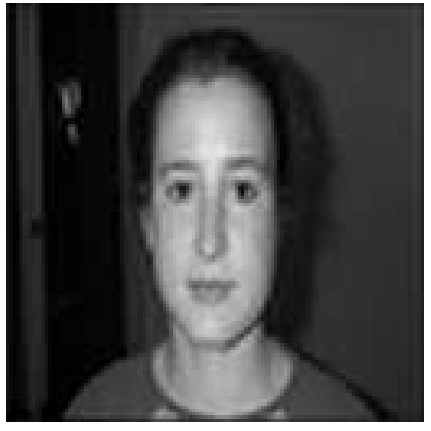

(b) UEP- $\Phi$ for whole image.

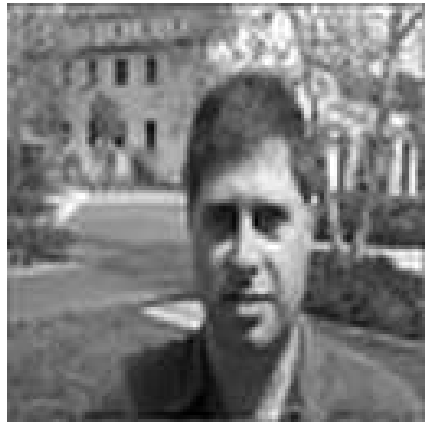

(e) UEP- $\Phi$ for whole image.

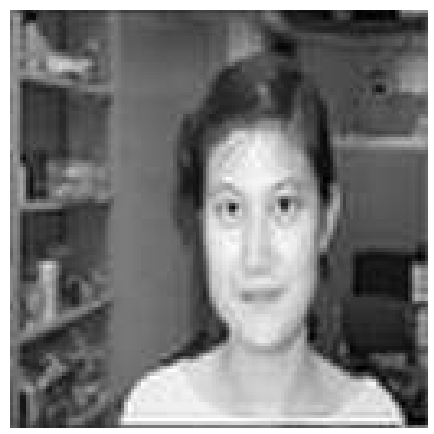

(h) UEP- $\Phi$ for whole image.

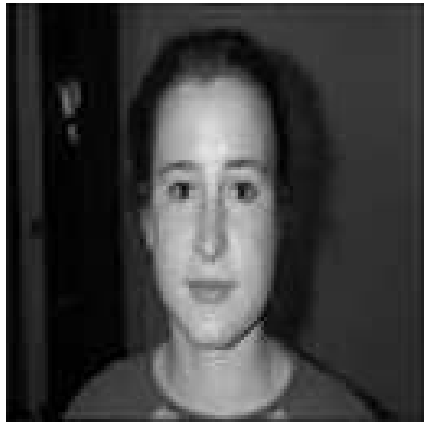

(c) UEP- $\Phi$ with prioritized ROI reconstruction.

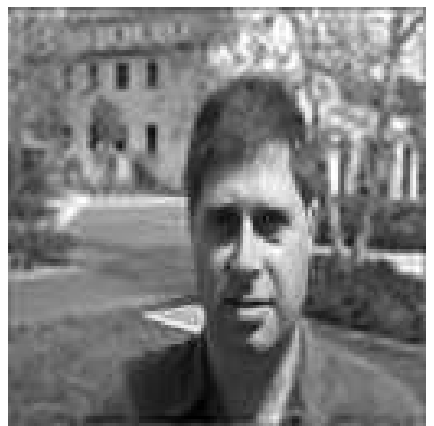

(f) UEP- $\Phi$ with prioritized ROI reconstruction.

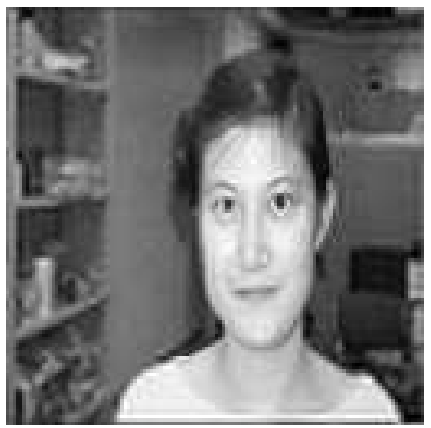

(i) UEP- $\Phi$ with prioritized ROI reconstruction.

Figure B.6: Visual performance comparison of various CS image reconstructions on sample images for $M=6000$. 


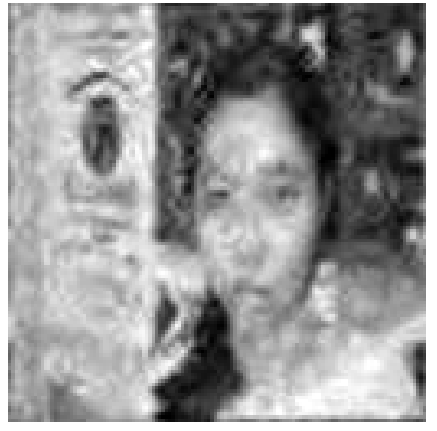

(a) TSWCS reconstruction.

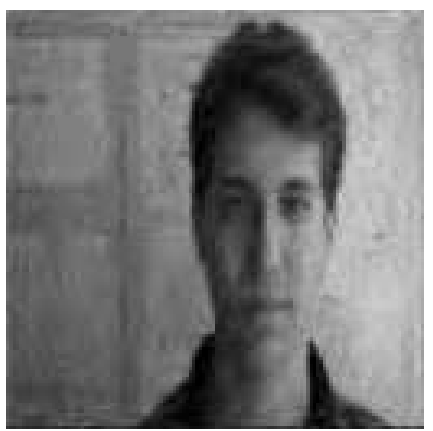

(d) TSWCS reconstruction.

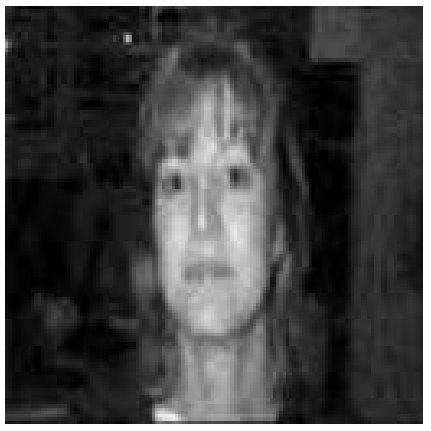

(g) TSWCS reconstruction.

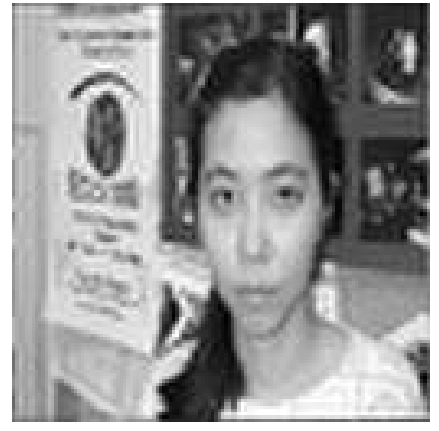

(b) UEP- $\Phi$ for whole image.

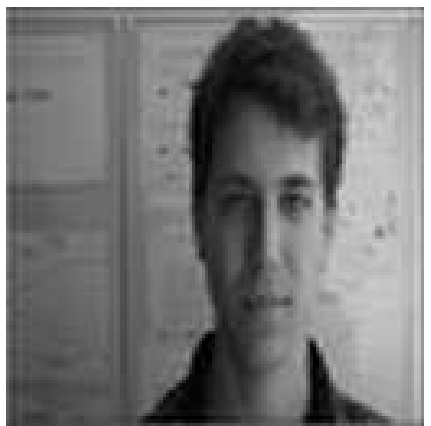

(e) UEP- $\Phi$ for whole image.

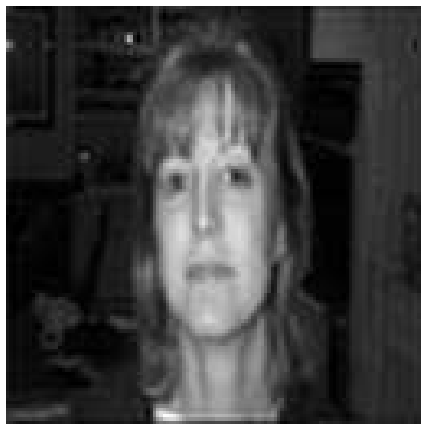

(h) UEP- $\Phi$ for whole image.

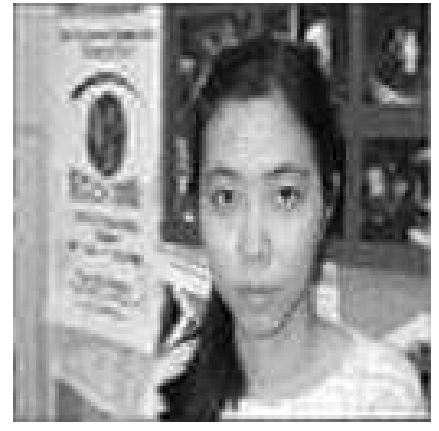

(c) UEP- $\Phi$ with prioritized ROI reconstruction.

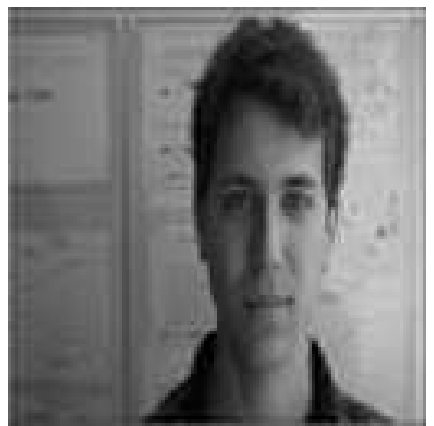

(f) UEP- $\Phi$ with prioritized ROI reconstruction.

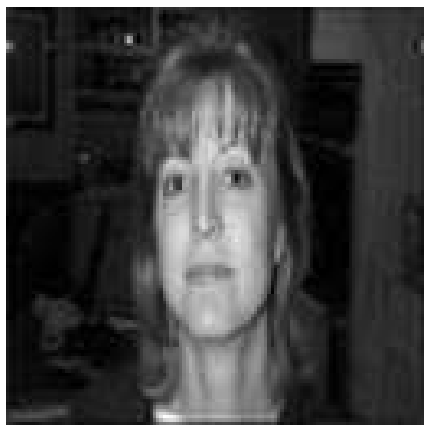

(i) UEP- $\Phi$ with prioritized ROI reconstruction.

Figure B.7: Visual performance comparison of various CS image reconstructions on sample images for $M=6000$. 
VITA

Betelhem Mateos Mekisso

Candidate for the Degree of

Master of Science

\section{Thesis: UNEQUAL COMPRESSIVE IMAGING}

Major Field: Electrical Engineering

Biographical:

Education:

Received the B.S. degree from Arba Minch University, Arba Minch, Ethiopia, in July 2007 , in Electrical Engineering

Completed the requirements for the degree of Master of Science with a major in Electrical Engineering Oklahoma State University in July, 2011.

Experience:

Worked as optical fiber and transmission engineer in Ethiopian Telecommunication Corporation, 2007 to 2008; Employed by Oklahoma State University, department of Electrical and Computer Engineering as a graduate research assistant, Oklahoma State University, department of Electrical and Computer Engineering, 2009 to 2010. 
Title of Study: UNEQUAL COMPRESSIVE IMAGING

Pages in Study: 62

Candidate for the Degree of Master of Science

Major Field: Electrical Engineering

In this thesis, we propose unequal compressive imaging for different compressive sensing scenarios. First, we consider the fact that a certain region of a given imagery is more important in most applications. Such a region in an image is known as the region of interest, e.g., the face in a portrait. We propose to capture the coefficients of the region of interest more strongly compared to the rest. With this setup, the region of interest is reconstructed with a higher accuracy, while the less important areas are slightly degraded.

Further, we consider the fact that in the the sparse representation of images the low frequency components (that convey a most of the image information) mostly appear at the beginning of the transform coefficients' vector. Therefore, we propose to capture the beginning transform coefficients by applying unequal compressive sampling, and show that this would significantly improve the quality of the reconstructed images.

Moreover, we exploit the structure of the wavelet tree to track the transform coefficients that correspond to the region of interest of images. We then propose an algorithm which strongly captures the low frequency coefficients and also those that correspond to the region of interest. With this setting, the overall quality of the reconstructed image is improved while the region of interest is reconstructed with an even better quality.

ADVISOR'S APPROVAL: 